\title{
Using Generalized Fibonacci Sequences for Solving the One-Dimensional LQR Problem and its Discrete-Time Riccati Equation
}

\author{
Johan Byström ${ }^{1}$ Lars Petter Lystad ${ }^{2}$ Per-Ole Nyman ${ }^{2}$ \\ ${ }^{1}$ Department of Mathematics, Luleå University of Technology, SE-97187 Luleå, Sweden. E-mail: johanb@ltu.se \\ ${ }^{2}$ Department of Technology, Narvik University College, NO-8505 Narvik, Norway. E-mail: \{lpl,pon\} @hin.no
}

\begin{abstract}
In this article we develop a method of solving general one-dimensional Linear Quadratic Regulator (LQR) problems in optimal control theory, using a generalized form of Fibonacci numbers. We find the solution $R(k)$ of the corresponding discrete-time Riccati equation in terms of ratios of generalized Fibonacci numbers. An explicit Binet type formula for $R(k)$ is also found, removing the need for recursively finding the solution at a given timestep. Moreover, we show that it is also possible to express the feedback gain, the penalty functional and the controller state in terms of these ratios. A generalized golden ratio appears in the corresponding infinite horizon problem. Finally, we show the use of the method in a few examples.
\end{abstract}

Keywords: LQR, Linear quadratic control, Optimal control, Fibonacci number, Golden ratio, Binet formula

\section{Introduction}

In the optimal control theory, one focuses on the problem of controlling a dynamic system under minimization of a penalty (or cost) functional. One of the most well-known optimal control problems is Linear Quadratic control, where the dynamic system is described by a set of linear differential equations, or linear difference equations, depending on whether continuous- or discrete-time is used. Moreover, the cost is described by a quadratic functional. In order to solve the discrete-time, finite horizon, linear quadratic control problem, typically one solves a nonlinear Riccati difference equation recursively. We refer to the books Kwakernaak and Sivan (1972) and Lewis and Syrmos (1995) for more information on optimal control theory.

The Italian matematician Leonardo of Pisa, known as Fibonacci, studied in the 13th century the growth of an idealised rabbit population and arrived in the nowadays famous Fibonacci sequence $\left(F_{n}\right)=$
$(0,1,1,2,3,5,8,13, \ldots)$, described by the recursive equation

$$
\begin{aligned}
F_{n} & =F_{n-1}+F_{n-2}, \\
F_{0} & =0, F_{1}=1 .
\end{aligned}
$$

These remarkable numbers have been shown to appear in such diverse fields as nature, art, geometry, architecture, music and even for calculating $\pi$. Moreover, one of the most fascinating facts is that the ratio $F_{n} / F_{n-1}$ of two consecutive numbers converge to the golden ratio (or golden section) $\varphi$ given by

$$
\varphi=\frac{1+\sqrt{5}}{2} \approx 1.618
$$

For more information regarding Fibonacci numbers and the golden ratio, we refer to The Fibonacci Association (http://www.mscs.dal.ca/Fibonacci) and its periodic publication The Fibonacci Quarterly (http://www.fq.math.ca). Other sources of interests 
are e.g. the books Dunlap (2003), Huntley (1970), Livio (2002) and Walser (2001).

There is in general not much known about the connection between optimal control and Fibonacci numbers. In Benavoli et al. (2009) relationships between the Fibonacci sequence and the Kalman filter gain, respectively its estimation error covariance, are derived for a simple plant model

$$
\begin{aligned}
& x(t+1)=x(t)+w(t), \\
& y(t+1)=x(t)+v(t),
\end{aligned}
$$

where $w$ and $v$ are noise terms of equal variance. It is shown that the steady-state Kalman gain and the estimation error covariance are then equal to the golden section and its conjugate, respectively.

For the more general case of a state equation

$$
x(t+1)=a x(t)+w(t)
$$

and non-equal noise variances, the authors show that two particular choices of a recover a steady-state Kalman filter gain equal to the golden section, respectively the conjugate golden section. Similar golden section filter gain situations are derived in Capponi et al. (2010) for the continuous-time Kalman filter and for the non-linear Benes filter.

Also somewhat related, it is shown in the article Lang (2004) that generalized Fibonacci numbers can be generated by a continuous-time Riccati differential equation.

In this article we investigate the relation between the one-dimensional linear quadratic control problem and Fibonacci numbers. More specific, we show that we can solve the corresponding discrete-time Riccati equation by introducing a generalized sequence of Fibonacci numbers and express different quantities in the LQR problem as ratios of generalized Fibonacci numbers. We also find explicit Binet type formulae based on these generalized Fibonacci numbers, removing the need to recursively find the solution at a given timestep. Finally, we show how to practically use the generalized Fibonacci sequences by numerically solving four linear quadratic examples in the last section.

\section{General Setting of a Finite Horizon Discrete-Time LQR}

Consider an $r$-dimensional discrete-time system

$$
\begin{aligned}
\mathbf{x}(k+1) & =\Phi \mathbf{x}(k)+\Gamma \mathbf{u}(k), k=0,1,2, \ldots, N,(1) \\
\mathbf{y}(k) & =\Delta \mathbf{x}(k),
\end{aligned}
$$

where $\mathbf{x}(\cdot) \in \mathcal{R}^{r}$ is the state vector, $\mathbf{u}(\cdot) \in \mathcal{R}^{s}$ the input vector, $\mathbf{y}(\cdot) \in \mathcal{R}^{t}$ the output vector, $\Phi \in \mathcal{R}^{r \times r}$ the state matrix, $\Gamma \in \mathcal{R}^{r \times s}$ the input matrix and $\Delta \in$ $\mathcal{R}^{t \times r}$ the output matrix. Moreover, we define the cost functional $J=J(\mathbf{x}, \mathbf{u})$ by

$$
J=\frac{1}{2} \sum_{m=0}^{N-1}\left[\mathbf{x}(m)^{T} Q \mathbf{x}(m)+\mathbf{u}(m)^{T} P \mathbf{u}(m)\right],
$$

where $P$ is a positive definite matrix and $Q$ is a positive semidefinite matrix. The cost functional is then minimized (see e.g. Åström and Wittenmark (1996)) by the optimal feedback control law

$$
\mathbf{u}(k)=-L(k) \mathbf{x}(k),
$$

where the feedback gain is given by

$$
L(k)=\left[\Gamma^{T} R(k+1) \Gamma+P\right]^{-1} \Gamma^{T} R(k+1) \Phi
$$

and $R(k)$ is the symmetric and positive semidefinite matrix that is the solution of the discrete-time Riccati difference equation

$$
\begin{aligned}
R(k)= & Q+\Phi^{T} R(k+1) \Phi-\Phi^{T} R(k+1) \Gamma \\
& {\left[\Gamma^{T} R(k+1) \Gamma+P\right]^{-1} \Gamma^{T} R(k+1) \Phi, } \\
R(N)= & 0 .
\end{aligned}
$$

This can be simplified as (see e.g. Balchen (1977))

$$
L(k)=P^{-1} \Gamma^{T} \Phi^{-T}(R(k)-Q),
$$

where $R(k)$ is the solution of the difference equation

$$
\begin{aligned}
R(k)= & Q+\Phi^{T} R(k+1)\left[I+\Gamma P^{-1} \Gamma^{T}\right. \\
& R(k+1)]^{-1} \Phi, k=N-1, \ldots, 1,0,(5) \\
R(N)= & 0 .
\end{aligned}
$$

We will show that this particular system in dimension $r=1$ has a special connection to a recurrence equation of Fibonacci type. For simplicity, we first consider the case when $\Phi^{2}=I=1$ and later generalize the result to any $\Phi$.

Remark 1 The above results are also true for a more general cost functional

$$
\begin{aligned}
& J=\frac{1}{2} \mathbf{x}(N)^{T} Q_{0} \mathbf{x}(N) \\
& +\frac{1}{2} \sum_{m=0}^{N-1}\left[\mathbf{x}(m)^{T} Q \mathbf{x}(m)+\mathbf{u}(m)^{T} P \mathbf{u}(m)\right]
\end{aligned}
$$

when the initial value $R(N)=0$ is replaced with $R(N)=Q_{0}$. It is also possible to include linear terms in the penalty functional by making appropriate transformations. 


\section{Generalized Fibonacci Sequences}

We define the generalized Fibonacci sequence $\left(f_{n}\right)_{n=0,1,2, \ldots}$ by the recurrence equation

$$
\begin{aligned}
f_{n} & =a f_{n-1}+b f_{n-2} \\
f_{0} & =0, f_{1}=1
\end{aligned}
$$

Remark 2 When $a$ and $b$ are integers, $f_{n}$ is also known as the Lucas sequence $U_{n}(a,-b)$, see e.g. Dickson (2002) or Ribenboim (2000). The special case $a=b=1$ gives the well known Fibonacci sequence $F_{n}=(0,1,1,2,3,5,8,13, \ldots)$, from hereon denoted by capital $F$.

We will from hereon assume that $b>0$ and $a \neq 0$ are real numbers, even though a lot of the results in this section easily can be generalized in the case of complex coefficients $a$ and $b$. However, due to the identification between these coefficients and the physical parameters arising from the LQR-problem, it is natural to use the imposed restrictions.

Moreover, we introduce the negative generalized Fibonacci numbers by

$$
f_{-n}=\frac{(-1)^{n+1}}{b^{n}} f_{n}
$$

which in particular gives $f_{-1}=1 / b$ and $f_{-2}=-a / b^{2}$. Depending on the values of $a$ and $b$, we have the following situations:

Theorem 3 Assume that we have defined the sequence $\left(f_{n}\right)_{n=0,1,2,3, \ldots}=\left(f_{0}, f_{1}, f_{2}, \ldots\right)$ as in (7) above. Then

1. $\left(\left|f_{n}\right|\right)$ will diverge to infinity as $n \rightarrow \infty$ if $|a|+b>$ 1 .

2. $\left(\left|f_{n}\right|\right)$ will converge to $\frac{1}{1+b}$ as $n \rightarrow \infty$ if $|a|+b=1$.

3. $\left(\left|f_{n}\right|\right)$ will converge to zero as $n \rightarrow \infty$ if $|a|+b<1$.

Proof. Cases (1) and (3) are obvious. For case (2) with $0<a<1$, consider the recurrence equation $f_{n}=$ $(1-b) f_{n-1}+b f_{n-2}, f_{0}=0, f_{1}=1$, where $0<b<1$. Then we have the two basis cases $f_{1}=1$ and $f_{2}=1-b$. Note that

$$
\lim _{n \rightarrow \infty} \sum_{k=0}^{n-1}(-1)^{k} b^{k}=\frac{1}{1+b} .
$$

Hence the proof is complete if we can show that $f_{n}=$ $\sum_{k=0}^{n-1}(-1)^{k} b^{k}$. Indeed, the assumptions

$$
f_{p}=\sum_{k=0}^{p-1}(-1)^{k} b^{k}
$$

and

$$
f_{p-1}=\sum_{k=0}^{p-2}(-1)^{k} b^{k}=f_{p}-(-1)^{p-1} b^{p-1},
$$

implies that

$$
\begin{aligned}
f_{p+1} & =(1-b) f_{p}+b f_{p-1} \\
& =(1-b) f_{p}+b\left(f_{p}-(-1)^{p-1} b^{p-1}\right) \\
& =f_{p}-(-1)^{p-1} b^{p}=f_{p}+(-1)^{p} b^{p} \\
& =\sum_{k=0}^{p}(-1)^{k} b^{k} .
\end{aligned}
$$

Thus the proof follows by the induction principle. In the same way, it easily follows in the case $-1<a<0$ that the $n^{\text {th }}$ generalized Fibonacci number is

$$
f_{n}=(-1)^{n-1} \sum_{k=0}^{n-1}(-1)^{k} b^{k}
$$

which also implies that

$$
\lim _{n \rightarrow \infty}\left|f_{n}\right|=\lim _{n \rightarrow \infty} \sum_{k=0}^{n-1}(-1)^{k} b^{k}=\frac{1}{1+b} .
$$

By using a so called Binet formula, it is possible to find the $n^{\text {th }}$ generalized Fibonacci number $f_{n}$ explicitly without the need for previous values. The formula is given in the following theorem:

Theorem 4 The $n^{\text {th }}$ generalized Fibonacci number $f_{n}$ is given by

$$
f_{n}=\frac{\left(\frac{a+\sqrt{a^{2}+4 b}}{2}\right)^{n}-\left(\frac{a-\sqrt{a^{2}+4 b}}{2}\right)^{n}}{\sqrt{a^{2}+4 b}} .
$$

Proof. Let us denote

$$
\varphi=\frac{a+\sqrt{a^{2}+4 b}}{2} .
$$

Then

$$
a-\varphi=\frac{a-\sqrt{a^{2}+4 b}}{2}=-\frac{b}{\varphi}
$$

This implies that $\varphi_{1}=\varphi$ is one solution of the characteristic equation

$$
\varphi^{2}=a \varphi+b
$$

and $\varphi_{2}=a-\varphi$ the other, since

$$
\begin{array}{r}
a-\varphi=-\frac{b}{\varphi} \Leftrightarrow \\
-b=(a-\varphi)[a-(a-\varphi)] \Leftrightarrow \\
(a-\varphi)^{2}=a(a-\varphi)+b .
\end{array}
$$


The difference between the solutions is then

$$
\varphi_{1}-\varphi_{2}=\varphi-(a-\varphi)=2 \varphi-a=\sqrt{a^{2}+4 b} .
$$

We thus need to prove the formula

$$
f_{n}=\frac{\varphi_{1}^{n}-\varphi_{2}^{n}}{\varphi_{1}-\varphi_{2}}=\frac{\varphi^{n}-(a-\varphi)^{n}}{2 \varphi-a} .
$$

It easily follows that

$$
\begin{aligned}
& f_{0}=\frac{\varphi^{0}-(a-\varphi)^{0}}{2 \varphi-a}=\frac{1-1}{2 \varphi-a}=0, \\
& f_{1}=\frac{\varphi^{1}-(a-\varphi)^{1}}{2 \varphi-a}=\frac{2 \varphi-a}{2 \varphi-a}=1 .
\end{aligned}
$$

Now assume that

$$
\begin{aligned}
f_{p-1} & =\frac{\varphi^{p-1}-(a-\varphi)^{p-1}}{2 \varphi-a}, \\
f_{p} & =\frac{\varphi^{p}-(a-\varphi)^{p}}{2 \varphi-a} .
\end{aligned}
$$

Then

$$
\begin{aligned}
f_{p+1}= & a f_{p}+b f_{p-1} \\
= & a \frac{\varphi^{p}-(a-\varphi)^{p}}{2 \varphi-a}+b \frac{\varphi^{p-1}-(a-\varphi)^{p-1}}{2 \varphi-a} \\
= & \frac{1}{2 \varphi-a}\left[(a \varphi+b) \varphi^{p-1}-\right. \\
& \left.(a(a-\varphi)+b)(a-\varphi)^{p-1}\right] \\
= & \frac{1}{2 \varphi-a}\left[\varphi^{2} \varphi^{p-1}-(a-\varphi)^{2}(a-\varphi)^{p-1}\right] \\
= & \frac{\varphi^{p+1}-(a-\varphi)^{p+1}}{2 \varphi-a} .
\end{aligned}
$$

Thus the proof follows by the induction principle.

Remark 5 The quantity $\varphi$ is the well known Golden ratio

$$
\varphi=\frac{1+\sqrt{5}}{2} \approx 1.618
$$

when $a=b=1$.

We define the sequence of Fibonacci ratios $\left(G_{n}\right)_{n=1,2,3, \ldots}$ by

$$
G_{n}=\frac{f_{n+1}}{f_{n}}
$$

We then find the consecutive elements $G_{n}$ by rewriting (7) as

$$
\frac{f_{n+1}}{f_{n}}=a+\frac{b}{\frac{f_{n}}{f_{n-1}}} \Leftrightarrow G_{n}=a+\frac{b}{G_{n-1}},
$$

or reversed

$$
G_{n-1}=\frac{b}{G_{n}-a},
$$

with initial value $G_{1}=a$. Clearly, $a>0$ implies that $G_{n}>0$ and $a<0$ implies that $G_{n}<0$ for all $n$, i.e.

$$
a G_{n}>0 .
$$

Therefore $a G_{n}=a^{2}+\frac{a^{2} b}{a G_{n-1}} \geq a^{2}$ and thus also $a G_{n}=$ $a^{2}+\frac{a^{2} b}{a G_{n-1}} \leq a^{2}+b$ for $n=1,2,3, \ldots$ Hence, it follows that

$$
a^{2} \leq a G_{n} \leq a^{2}+b,
$$

with equality only for $n=1$ and $n=2$, respectively. In a similar fashion, it follows that the reciprocal sequence $\left(H_{n}\right)_{n=1,2,3, \ldots}$ defined by

$$
\begin{aligned}
& H_{n}=\frac{f_{n-1}}{f_{n}}, \\
& H_{1}=0,
\end{aligned}
$$

can be generated by the recursive relation

$$
H_{n}=\frac{1}{a+b H_{n-1}}
$$

and is limited by

$$
\frac{1}{a^{2}+b} \leq \frac{H_{n}}{a} \leq \frac{1}{a^{2}}
$$

for $n=2,3,4, \ldots$, with equality only for $n=2$ and $n=3$, respectively.

As a consequence of the Binet formula (9), we get explicit expressions for $G_{n}$ and $H_{n}$ by the formulae

$$
\begin{aligned}
& G_{n}=\frac{\left(\frac{a+\sqrt{a^{2}+4 b}}{2}\right)^{n+1}-\left(\frac{a-\sqrt{a^{2}+4 b}}{2}\right)^{n+1}}{\left(\frac{a+\sqrt{a^{2}+4 b}}{2}\right)^{n}-\left(\frac{a-\sqrt{a^{2}+4 b}}{2}\right)^{n}}, \\
& H_{n}=\frac{\left(\frac{a+\sqrt{a^{2}+4 b}}{2}\right)^{n-1}-\left(\frac{a-\sqrt{a^{2}+4 b}}{2}\right)^{n-1}}{\left(\frac{a+\sqrt{a^{2}+4 b}}{2}\right)^{n}-\left(\frac{a-\sqrt{a^{2}+4 b}}{2}\right)^{n}} .
\end{aligned}
$$

Theorem 6 Both $\left(G_{n}\right)$ and $\left(H_{n}\right)$ are Cauchy sequences.

Proof. The identity

$$
\begin{aligned}
& \left(G_{n+1}-G_{n}\right)^{2}-\left(\frac{b}{G_{n} G_{n-1}}\right)^{2}\left(G_{n}-G_{n-1}\right)^{2} \\
= & \left(a+\frac{b}{G_{n}}-G_{n}\right)^{2}-\left(\frac{G_{n}-a}{G_{n}}\right)^{2}\left(G_{n}-\frac{b}{G_{n}-a}\right)^{2} \\
= & \frac{\left(a G_{n}+b-G_{n}^{2}\right)^{2}-\left(G_{n}\left(G_{n}-a\right)-b\right)^{2}}{G_{n}^{2}}=0
\end{aligned}
$$


implies that

$$
\left|G_{n+1}-G_{n}\right|=\left(\frac{b}{G_{n} G_{n-1}}\right)\left|G_{n}-G_{n-1}\right|,
$$

for $n=2,3,4, \ldots$ By rewriting the multiplicative contraction factor as

$$
K_{n}=\frac{b}{G_{n} G_{n-1}}=\frac{G_{n}-a}{G_{n}}=1-\frac{a^{2}}{a G_{n}}
$$

and using the fact that $a^{2}<a G_{n} \leq a^{2}+b$, we see that

$$
0<K_{n} \leq \frac{b}{a^{2}+b}<1 .
$$

Thus the difference between consecutive elements in $\left(G_{n}\right)$ becomes smaller and smaller. By an analogous derivation it is possible to show that

$$
\left|H_{n+1}-H_{n}\right|=b H_{n} H_{n+1}\left|H_{n}-H_{n-1}\right|
$$

for $n=2,3,4, \ldots$, where the multiplicative factor is

$$
k_{n}=b H_{n} H_{n+1}=1-a^{2} \frac{H_{n+1}}{a} .
$$

Therefore also $0<k_{n} \leq \frac{b}{a^{2}+b}<1$, since $\frac{1}{a^{2}+b} \leq \frac{H_{n}}{a}<$ $\frac{1}{a^{2}}$. It thus follows that both sequences are Cauchy.

Corollary 7 Hence the sequences $\left(G_{n}\right)$ and $\left(H_{n}\right)$ converge to the unique and finite limits

$$
\begin{aligned}
& G_{\infty}=\lim _{n \rightarrow \infty} G_{n}=\frac{a^{2}+\sqrt{a^{4}+4 a^{2} b}}{2 a}, \\
& H_{\infty}=\lim _{n \rightarrow \infty} H_{n}=\frac{\sqrt{a^{4}+4 a^{2} b}-a^{2}}{2 a b}=\frac{1}{G_{\infty}} .
\end{aligned}
$$

Proof. The sequences are convergent since they are Cauchy. The limit for $\left(G_{n}\right)$ is found by letting $n \rightarrow \infty$ in equation (11) above, which yields

$$
\begin{aligned}
G_{\infty} & =a+\frac{b}{G_{\infty}} \\
& \mathbb{} \\
G_{\infty}^{2}-a G_{\infty}-b & =0 \Leftrightarrow G_{\infty}=\frac{a \pm \sqrt{a^{2}+4 b}}{2} .
\end{aligned}
$$

Obviously $\sqrt{a^{2}+4 b}>|a|$. Therefore, since all elements $G_{n}$ are positive if $a>0$ and negative if $a<0$, we conclude that the limit must be

$$
G_{\infty}= \pm \frac{|a|+\sqrt{a^{2}+4 b}}{2}=\frac{a^{2}+\sqrt{a^{4}+4 a^{2} b}}{2 a},
$$

where the positive sign is chosen if $a>0$ and the negative sign is chosen if $a<0$. The corresponding proof for $\left(H_{n}\right)$ follows analogously from equation (12).
The sequence $\left(G_{n}\right)$ oscillates around $G_{\infty}$ with consecutive elements being above and below $G_{\infty}$, since if $a G_{n-1}<a G_{\infty}$, we get that

$$
a G_{n}=a^{2}+\frac{a^{2} b}{a G_{n-1}}>a^{2}+\frac{a^{2} b}{a G_{\infty}}=a G_{\infty}
$$

and vice versa. The same is true for the sequence $\left(H_{n}\right)$, since $\frac{H_{n-1}}{a}<\frac{H_{\infty}}{a}$ implies that

$$
\frac{H_{n}}{a}=\frac{1}{a^{2}+a^{2} b \frac{H_{n-1}}{a}}>\frac{1}{a^{2}+a^{2} b \frac{H_{\infty}}{a}}=\frac{H_{\infty}}{a} .
$$

The rate of convergence to the corresponding limit elements follows from the identities

$$
\begin{aligned}
\left|G_{n}-G_{\infty}\right| & =\frac{b}{G_{\infty} G_{n-1}}\left|G_{n-1}-G_{\infty}\right|, \\
\left|H_{n}-H_{\infty}\right| & =b H_{n} H_{\infty}\left|H_{n-1}-H_{\infty}\right|,
\end{aligned}
$$

since

$$
\begin{aligned}
& \left(G_{n}-G_{\infty}\right)^{2}-\frac{b^{2}}{G_{\infty}^{2} G_{n-1}^{2}}\left(G_{n-1}-G_{\infty}\right)^{2} \\
= & \frac{\left(a+\frac{b}{G_{n-1}}-G_{\infty}\right)^{2} G_{n-1}^{2}-\frac{b^{2}}{G_{\infty}^{2}}\left(G_{n-1}-G_{\infty}\right)^{2}}{G_{n-1}^{2}} \\
= & \frac{\left(a G_{n-1}+b-G_{\infty} G_{n-1}\right)^{2}-\left(\frac{b}{G_{\infty}} G_{n-1}-b\right)^{2}}{G_{n-1}^{2}} \\
= & \frac{\left(a G_{n-1}+b-G_{\infty} G_{n-1}-\frac{b}{G_{\infty}} G_{n-1}+b\right)}{G_{n-1}} \\
= & \frac{\left(a G_{n-1}+b-G_{\infty} G_{n-1}+\frac{b}{G_{\infty}} G_{n-1}-b\right)}{G_{n-1}} \\
= & 0,
\end{aligned}
$$

where the last equality follows from (15).

Moreover, since $G_{n-1}$ converges to $G_{\infty}$, we have for sufficiently large $n$ that the constant of contraction is approximately

$$
\begin{aligned}
& \frac{b}{G_{\infty} G_{n-1}} \approx \frac{b}{G_{\infty}^{2}}=\frac{4 b}{\left(|a|+\sqrt{a^{2}+4 b}\right)^{2}} \\
& <\frac{4 b}{2 a^{2}+4 b}=\frac{1}{1+\frac{a^{2}}{2 b}}<1 .
\end{aligned}
$$

A similar calculation shows that the corresponding result is also true for the sequence $\left(H_{n}\right)$, with the constant of contraction again being

$$
b H_{n} H_{\infty} \approx b H_{\infty}^{2}=\frac{b}{G_{\infty}^{2}}<\frac{1}{1+\frac{a^{2}}{2 b}}<1 .
$$


Since consecutive elements of $\left(G_{n}\right)$ oscillate around the limit $G_{\infty}$, it follows that the subsequences with oddindexed and even-indexed elements of $\left(G_{n}\right)$ must have all elements either above or below the limit. Now

$$
a G_{1}=a^{2}<\frac{a^{2}+\sqrt{a^{4}+4 a^{2} b}}{2}=a G_{\infty},
$$

hence the elements of $\left(a G_{n}\right)_{n=1,3,5, \ldots}$ all lie below $a G_{\infty}$ and the elements of $\left(a G_{n}\right)_{n=2,4,6, \ldots}$ all lie above $a G_{\infty}$. Similarly, all elements of $\left(H_{n} / a\right)_{n=1,3,5, \ldots}$ lie below $H_{\infty} / a$ and all elements of $\left(H_{n} / a\right)_{n=0,2,4, \ldots}$ lie above $H_{\infty} / a$.

For this particular problem, we are only interested in the odd-indexed subsequences $G_{n}^{o}=G_{2 n-1}$ and $H_{n}^{o}=$ $H_{2 n-1}$. By using the recurrence formula twice, we can find expressions for the elements of these subsequences. Indeed,

$$
\begin{aligned}
G_{n+1} & =a+b / G_{n} \\
G_{n+2} & =a+b / G_{n+1}
\end{aligned}
$$

yields that

$$
\begin{aligned}
G_{n+2} & =a+\frac{b}{a+\frac{b}{G_{n}}}=\frac{\left(a^{2}+b\right) G_{n}+a b}{a G_{n}+b} \\
G_{1} & =a .
\end{aligned}
$$

Similarly, we find the corresponding recurrence equation for the odd-indexed subsequence of $\left(H_{n}\right)$ to be

$$
\begin{aligned}
H_{n+2} & =\frac{1}{a+b \frac{1}{a+b H_{n}}}=\frac{a+b H_{n}}{a^{2}+b+a b H_{n}}, \\
H_{1} & =0 .
\end{aligned}
$$

Alternatively, with use of the negative generalized Fibonacci numbers (8) we might also include the first negative indexed elements

$$
\begin{aligned}
G_{-1} & =\frac{f_{0}}{f_{-1}}=0, \\
H_{-1} & =\frac{f_{-2}}{f_{-1}}=-\frac{a}{b},
\end{aligned}
$$

as initial values for the recurrence equations.

Theorem 8 The odd-indexed and even-indexed subsequences of $\left(G_{n}\right)$ and $\left(H_{n}\right)$ are monotone.

Proof. Consider for instance the subsequence $\left(G_{n}\right)_{n=1,3,5, \ldots}$. Then $a^{2} \leq a G_{n}<a G_{\infty}, n=1,3,5, \ldots$, which implies that

$$
\begin{aligned}
& a^{2}\left(G_{n}^{2}-a G_{n}-b\right)=a G_{n}\left(a G_{n}-a^{2}\right)-a^{2} b \\
& <a G_{\infty}\left(a G_{\infty}-a^{2}\right)-a^{2} b \\
& =a^{2}\left(G_{\infty}^{2}-a G_{\infty}-b\right)=0 .
\end{aligned}
$$

Hence

$$
\begin{aligned}
a G_{n+2}-a G_{n} & =a \frac{\left(a^{2}+b\right) G_{n}+a b}{a G_{n}+b}-a G_{n} \\
& =-\frac{a^{2}\left(G_{n}^{2}-a G_{n}-b\right)}{G_{n} G_{n+1}}>0
\end{aligned}
$$

which implies that the subsequence $\left(a G_{n}\right)_{n=1,3,5, \ldots}$ is monotonically increasing. Similar arguments show that also $\left(\frac{H_{n}}{a}\right)_{n=1,3,5, \ldots}$ is monotonically increasing.

\section{A Special One-Dimensional LQR Problem}

In this section, we deal with the special case $\Phi^{2}=1$ for the LQR problem (1) in dimension $r=1$. We thus consider the optimal feedback control of the system

$$
\begin{aligned}
x(k+1) & =\Phi x(k)+\Gamma u(k), \\
y(k) & =\Delta x(k),
\end{aligned}
$$

subject to minimization of the functional

$$
J=\frac{1}{2} \sum_{m=0}^{N-1} Q(x(m))^{2}+P(u(m))^{2},
$$

where $Q \geq 0$ and $P>0$.

Remark 9 The degenerate case $Q=0$ gives the explicit solutions

$$
\begin{aligned}
R(N-k) & =\frac{P Q_{0} \Phi^{2 k}}{P+\Gamma^{2} Q_{0} \sum_{i=0}^{k-1} \Phi^{2 i}}=\frac{P Q_{0}}{P+k \Gamma^{2} Q_{0}} \\
L(N-k) & =\frac{\Gamma}{P \Phi} R(N-k)=\frac{\Gamma Q_{0} / \Phi}{P+k \Gamma^{2} Q_{0}}
\end{aligned}
$$

for $k=1,2, \ldots, N$, where $Q_{0}$ is taken from (6). Specifically, we have that $R(k)=0$ and $L(k)=0$ for all $k$ when $Q_{0}=0$. We therefore only consider the case $Q>0$ from hereon.

To ensure controllability of the system, we also require $\Gamma \neq 0$. Then the corresponding Riccati difference equation (5) becomes

$$
\begin{aligned}
R(k)= & Q+\frac{\Phi^{2} P R(k+1)}{P+\Gamma^{2} R(k+1)}, \\
& k=N-1, \ldots, 1,0, \\
R(N)= & 0 .
\end{aligned}
$$

To begin with, we note that this implies that

$$
R(N-1)=Q
$$


The feedback control gain (4) then becomes

$$
L(k)=\frac{\Gamma(R(k)-Q)}{\Phi P}=\frac{\Phi \Gamma R(k+1)}{P+\Gamma^{2} R(k+1)} .
$$

Inverting this relation yields

$$
R(k)=\frac{P \Phi}{\Gamma} L(k)+Q,
$$

hence

$$
R(k+1)=\frac{P \Phi}{\Gamma} L(k+1)+Q
$$

Replacing $R(k+1)$ in $(21)$ with $(22)$ gives the corresponding recurrence equation for the feedback gain as

$$
\begin{aligned}
L(k) & =\frac{\Gamma \Phi\left(\frac{P \Phi}{\Gamma} L(k+1)+Q\right)}{P+\Gamma^{2}\left(\frac{P \Phi}{\Gamma} L(k+1)+Q\right)} \\
& =\frac{\Gamma \Phi+\frac{P}{Q} L(k+1) \Phi^{2}}{\Gamma^{2}+\frac{P}{Q}+\Gamma \frac{P}{Q} \Phi L(k+1)},
\end{aligned}
$$

with the initial value

$$
L(N)=\Gamma \frac{R(N)-Q}{\Phi P}=-\frac{Q}{\Phi P} \Gamma .
$$

If we divide $L(k)$ with $\Phi$, we get the equivalent recurrence equation

$$
\frac{L(k)}{\Phi}=\frac{\Gamma+\frac{P}{Q} \Phi^{2} \frac{L(k+1)}{\Phi}}{\Gamma^{2}+\frac{P}{Q}+\Gamma \frac{P}{Q} \Phi^{2} \frac{L(k+1)}{\Phi}}, k=N-1, \ldots, 1,0,
$$

in the variable $L(\cdot) / \Phi$, with the initial value

$$
\frac{L(N)}{\Phi}=-\frac{Q}{P \Phi^{2}} \Gamma
$$

However, since $R(N-1)=Q$, we can also use

$$
\frac{L(N-1)}{\Phi}=\Gamma \frac{R(N-1)-Q}{\Phi^{2} P}=0
$$

as initial value. Moreover, by multiplying (20) with $\Gamma / Q$, we get an equivalent difference equation in the variable $\frac{\Gamma}{Q} R(\cdot)$ by

$$
\begin{aligned}
\frac{\Gamma}{Q} R(k) & =\Gamma+\frac{P \frac{\Gamma}{Q} \Phi^{2} R(k+1)}{P+\Gamma^{2} R(k+1)} \\
& =\frac{\Gamma \frac{P}{Q}+\left(\Gamma^{2}+\frac{P}{Q} \Phi^{2}\right) \frac{\Gamma}{Q} R(k+1)}{\frac{P}{Q}+\Gamma \frac{\Gamma}{Q} R(k+1)} \\
\frac{\Gamma}{Q} R(N) & =0 .
\end{aligned}
$$

We can here alternatively use

$$
\frac{\Gamma}{Q} R(N-1)=\Gamma
$$

as initial value. This leads us to the theorem:
Theorem 10 Assume that $\Phi^{2}=1$ and let $f_{n}$ be the generalized Fibonacci numbers generated by

$$
\begin{aligned}
f_{n} & =\Gamma f_{n-1}+\frac{P}{Q} f_{n-2}, \\
f_{0} & =0, f_{1}=1 .
\end{aligned}
$$

Then the solution $R(N-k), k=1,2, \ldots, N$, of the Riccati equation (20) is given by

$$
R(N-k)=\frac{Q}{\Gamma} G_{2 k-1}=\frac{Q}{\Gamma} \frac{f_{2 k}}{f_{2 k-1}},
$$

and the feedback gain $L(N-k)$ is given by

$$
L(N-k)=\Phi H_{2 k-1}=\Phi \frac{f_{2(k-1)}}{f_{2 k-1}} .
$$

Proof. Consider the definition (7) with

$$
\begin{aligned}
a & =\Gamma, \\
b & =\frac{P}{Q} .
\end{aligned}
$$

Then the recurrence equation (17) for the odd-indexed subsequence of $\left(G_{n}\right)$ becomes

$$
\begin{aligned}
G_{n+2} & =\frac{\left(\Gamma^{2}+\frac{P}{Q}\right) G_{n}+\Gamma \frac{P}{Q}}{\Gamma G_{n}+\frac{P}{Q}}, n=-1,1,3,5, \ldots, \\
G_{-1} & =0 .
\end{aligned}
$$

If we do not want to use negative-indexed values, we can replace the initial value with $G_{1}=a=\Gamma$. By making the change of variable

$$
n=2(N-k)-1,
$$

we see that this is exactly the same recurrence equation with same initial value as equation (24) for $\frac{\Gamma}{Q} R(k)$, when $\Phi^{2}=1$. By uniqueness of the solution of this kind of recurrence equations (function iteration), we find that

$$
\frac{\Gamma}{Q} R(k)=G_{2(N-k)-1}=\frac{f_{2(N-k)}}{f_{2(N-k)-1}},
$$

that is

$$
R(N-k)=\frac{Q}{\Gamma} G_{2 k-1}=\frac{Q}{\Gamma} \frac{f_{2 k}}{f_{2 k-1}} .
$$

Similarly, we get that the recurrence equation (18) for the odd-indexed subsequence of $\left(H_{n}\right)$ becomes

$$
\begin{aligned}
H_{n+2} & =\frac{\Gamma+\frac{P}{Q} H_{n}}{\Gamma^{2}+\frac{P}{Q}+\Gamma \frac{P}{Q} H_{n}}, \\
H_{-1} & =-\frac{Q}{P} \Gamma .
\end{aligned}
$$


If we do not want to use negative-indexed values, we can replace the initial value with $H_{1}=0$. Using $\Phi^{2}=$ 1 , we note that this is exactly the same recurrence equation with same initial value as equation (23) for $L(k) / \Phi$, where the relationship between indices $n$ and $k$ again is

$$
n=2(N-k)-1 .
$$

Hence we find that

$$
\frac{L(k)}{\Phi}=H_{2(N-k)-1}=\frac{f_{2(N-k-1)}}{f_{2(N-k)-1}},
$$

which implies that

$$
L(N-k)=\Phi H_{2 k-1}=\Phi \frac{f_{2(k-1)}}{f_{2 k-1}} .
$$

Knowing the feedback gain, we can now compute the state and input sequences. The results are contained in the following theorem:

Theorem 11 Given the initial value $x(0)$, we can express the state and input values $x(k)$ and $u(k)$, $k=0,1,2, \ldots, N-1$, in the system (19) as

$$
\begin{aligned}
& x(k)=\frac{f_{2(N-k)-1}}{f_{2 N-1}}\left(\Phi \frac{P}{Q}\right)^{k} x(0), \\
& u(k)=-\Phi \frac{f_{2(N-k-1)}}{f_{2 N-1}}\left(\Phi \frac{P}{Q}\right)^{k} x(0) .
\end{aligned}
$$

Proof. If the formula for $x(k)$ is true, it easily follows from (27) that

$$
\begin{aligned}
u(k) & =-L(k) x(k) \\
& =-\Phi \frac{f_{2(N-k-1)}}{f_{2(N-k)-1}} \frac{f_{2(N-k)-1}}{f_{2 N-1}}\left(\Phi \frac{P}{Q}\right)^{k} x(0) \\
& =-\Phi \frac{f_{2(N-k-1)}}{f_{2 N-1}}\left(\Phi \frac{P}{Q}\right)^{k} x(0) .
\end{aligned}
$$

First, we note that the formula for $x(k)$ is trivially true when $k=0$. Now assume that the formula is true for $k=p$, i.e.

$$
x(p)=\frac{f_{2(N-p)-1}}{f_{2 N-1}}\left(\Phi \frac{P}{Q}\right)^{p} x(0) .
$$

Then

$$
\begin{aligned}
x(p+1)= & \Phi x(p)+\Gamma u(p)=(\Phi-\Gamma L(p)) x(p) \\
= & \left(\Phi-\Gamma \Phi \frac{f_{2(N-p-1)}}{f_{2(N-p)-1}}\right) x(p) \\
= & \Phi \frac{f_{2(N-p)-1}-\Gamma f_{2(N-p-1)}}{f_{2(N-p)-1}} \\
& \frac{f_{2(N-p)-1}\left(\Phi \frac{P}{Q}\right)^{p} x(0)}{f_{2 N-1}}\left(\Phi \frac{P}{Q}\right)^{p+1} x(0), \\
= & \frac{f_{2(N-(p+1))-1}}{f_{2 N-1}}(x)
\end{aligned}
$$

where the last equality follows from the definition of generalized Fibonacci numbers

$$
f_{m}-\Gamma f_{m-1}=\frac{P}{Q} f_{m-2}
$$

with $m=2(N-p)-1$ (odd number). The induction principle completes the proof.

Moreover, we have the following result for the subsequent values of the penalty functional:

Theorem 12 Let us define the value $J_{k}$ at timestep $k$ of the penalty functional by

$$
J_{k}=\frac{1}{2} \sum_{m=k}^{N-1} Q(x(m))^{2}+P(u(m))^{2} .
$$

Then the subsequent values $J_{k}, k=N-1, N-2, \ldots, 0$, are given by

$$
\begin{aligned}
J_{k} & =\frac{1}{2} R(k)(x(k))^{2}=\frac{Q(x(k))^{2}}{2 \Gamma} \frac{f_{2(N-k)}}{f_{2(N-k)-1}} \\
& =\frac{Q}{2 \Gamma}\left(\Phi \frac{P}{Q}\right)^{2 k} \frac{f_{2(N-k)} f_{2(N-k)-1}}{\left(f_{2 N-1}\right)^{2}}(x(0))^{2}
\end{aligned}
$$

Proof. From (21) we have that

$$
\Phi P L(k)+\Gamma Q-\Gamma R(k)=0 .
$$

Moreover, inversion of (20) yields that

$$
R(k+1)=\frac{P(R(k)-Q)}{\Phi^{2} P+Q \Gamma^{2}-\Gamma^{2} R(k)} .
$$

When $m=N-1$, we trivially have that

$$
\begin{aligned}
& J_{N-1}=\frac{1}{2} Q(x(N-1))^{2}=\frac{1}{2} R(N-1)(x(N-1))^{2}, \\
& \text { since } R(N-1)=Q \text { and } u(N-1)= \\
& -L(N-1) x(N-1)=0 . \text { Let us now assume } \\
& \text { that (31) holds for } k=p+1, \text { i.e. }
\end{aligned}
$$

$$
\begin{aligned}
J_{p+1} & =\frac{1}{2} R(p+1)(x(p+1))^{2} \\
& =\frac{1}{2} R(p+1)(\Phi x(p)+\Gamma u(p))^{2} .
\end{aligned}
$$


Then it follows that

$$
\begin{aligned}
J_{p}= & J_{p+1}+\frac{1}{2}\left(Q(x(p))^{2}+P(u(p))^{2}\right) \\
= & \frac{1}{2}\left[R(p+1)(\Phi x(p)+\Gamma u(p))^{2}+Q(x(p))^{2}\right. \\
& \left.+P(u(p))^{2}\right] \\
= & \frac{1}{2}\left[\frac{P(R(p)-Q)(\Phi-\Gamma L(p))^{2}}{\Phi^{2} P+Q \Gamma^{2}-\Gamma^{2} R(p)}+Q\right. \\
& \left.+P(L(p))^{2}\right](x(p))^{2} \\
= & \frac{1}{2} \frac{1}{\Phi^{2} P+Q \Gamma^{2}-\Gamma^{2} R(p)}[(\Phi P L(p)+\Gamma Q \\
& \left.-\Gamma R(p))^{2}+R(p)\left(\Phi^{2} P+Q \Gamma^{2}-\Gamma^{2} R(p)\right)\right] \\
& (x(p))^{2} \\
= & \frac{1}{2} R(p)(x(p))^{2} .
\end{aligned}
$$

Using the induction principle concludes the first part of (31). Secondly, using (26) and (28), we find that the consecutive values $J_{k}$ of the penalty functional can be expressed as

$$
\begin{aligned}
J_{k} & =\frac{1}{2} R(k)(x(k))^{2}=\frac{Q(x(k))^{2}}{2 \Gamma} \frac{f_{2(N-k)}}{f_{2(N-k)-1}} \\
& =\frac{Q}{2 \Gamma}\left(\Phi \frac{P}{Q}\right)^{2 k} \frac{f_{2(N-k)} f_{2(N-k)-1}}{\left(f_{2 N-1}\right)^{2}}(x(0))^{2}
\end{aligned}
$$

Remark 13 Let $\Phi^{2}=1$. By first using definition (30) together with expressions (28) and (29) to compute $J_{0}$ and then comparing with the result

$$
J=J_{0}=\frac{Q}{2 \Gamma} \frac{f_{2 N}}{f_{2 N-1}}(x(0))^{2}
$$

from formula (31), one obtains the identity

$$
\sum_{m=0}^{M}\left(\frac{Q}{P}\right)^{m} f_{m}^{2}=\left(\frac{Q}{P}\right)^{M} \frac{f_{M} f_{M+1}}{\Gamma}
$$

(for $M=2 N-1$ odd, but it is also true for $M$ even), which in the case $\Gamma=\frac{P}{Q}=1$ simplifies to the well known Fibonacci identity

$$
\sum_{m=0}^{M} F_{m}^{2}=F_{M} F_{M+1}
$$

Moreover, by using (34) we see that

$$
\begin{aligned}
\lim _{N \rightarrow \infty} J & =\lim _{N \rightarrow \infty} \frac{1}{2} \sum_{m=0}^{N-1} Q(x(m))^{2}+P(u(m))^{2} \\
& =\frac{Q}{2 \Gamma} G_{\infty}(x(0))^{2} .
\end{aligned}
$$

By using the explicit Binet type formulae (13) and (14), we may also conclude that

$$
\begin{aligned}
& R(N-k)= \\
& \frac{Q}{\Gamma} \frac{\left(\frac{\Gamma+\sqrt{\Gamma^{2}+4 \frac{P}{Q}}}{2}\right)^{2 k}-\left(\frac{\Gamma-\sqrt{\Gamma^{2}+4 \frac{P}{Q}}}{2}\right)^{2 k}}{\left(\frac{\Gamma+\sqrt{\Gamma^{2}+4 \frac{P}{Q}}}{2}\right)^{2 k-1}-\left(\frac{\Gamma-\sqrt{\Gamma^{2}+4 \frac{P}{Q}}}{2}\right)^{2 k-1}}, \\
& L(N-k)= \\
& \Phi \frac{\left(\frac{\Gamma+\sqrt{\Gamma^{2}+4 \frac{P}{Q}}}{2}\right)^{2(k-1)}-\left(\frac{\Gamma-\sqrt{\Gamma^{2}+4 \frac{P}{Q}}}{2}\right)^{2(k-1)}}{\left(\frac{\Gamma+\sqrt{\Gamma^{2}+4 \frac{P}{Q}}}{2}\right)^{2 k-1}-\left(\frac{\Gamma-\sqrt{\Gamma^{2}+4 \frac{P}{Q}}}{2}\right)^{2 k-1}} .
\end{aligned}
$$

Corresponding Binet type formulae for $x(k), u(k)$ and $J_{k}$ may obviously be found in an analogous way.

Now consider the case $\Gamma>0$. It is obvious that $(R(N-k))_{k}$ is monotonically increasing (as $k$ increases from $k=0$ to $k=N$ ), since the corresponding generalized Fibonacci ratios are monotonically increasing by Theorem 8 . More specific, in the case with infinite horizon (i.e. $N \rightarrow \infty$ ), we have that $R_{\infty}(k)$ and $L_{\infty}(k)$ converge to the limit values

$$
\begin{aligned}
& R_{\infty}(0)=\lim _{k \rightarrow 0} R_{\infty}(k)=\frac{Q}{\Gamma} G_{\infty}=\frac{Q}{\Gamma} \varphi, \\
& L_{\infty}(0)=\lim _{k \rightarrow 0} L_{\infty}(k)=\Phi H_{\infty}=\Phi \frac{1}{\varphi},
\end{aligned}
$$

where subscript $\infty$ denotes infinite horizon and

$$
\varphi=G_{\infty}=\frac{\Gamma+\sqrt{\Gamma^{2}+4 \frac{P}{Q}}}{2} .
$$

The case $\Gamma<0$ follows analogously with the corresponding sign corrections.

Remark 14 The more general cost functional (6) leads to the same conclusions as above, however with the initial conditions $f_{0}$ and $f_{1}$ of the generalized $F i$ bonacci recurrence equation (25) replaced with

$$
\begin{aligned}
f_{0} & =\frac{\Gamma}{P} Q_{0}, \\
f_{1} & =1+\frac{\Gamma^{2}}{P} Q_{0},
\end{aligned}
$$

due to the change of initial value $R(N)=Q_{0}$. In this case we also have to redefine negative-indexed generalized Fibonacci numbers accordingly, since $f_{0} \neq 0$. Thus we obtain

$$
\begin{aligned}
& f_{-1}=\frac{Q}{P}\left(f_{1}-\Gamma f_{0}\right)=\frac{Q}{P} \\
& f_{-2}=\left(\frac{Q}{P}\right)^{2}\left(\left(\Gamma^{2}+\frac{P}{Q}\right) f_{0}-\Gamma f_{1}\right)=\frac{\Gamma Q}{P^{2}}\left(Q_{0}-Q\right) .
\end{aligned}
$$




\section{The General One-Dimensional Case}

Let us now consider the case without restrictions on $\Phi$, i.e. the optimal feedback control of the system

$$
\begin{aligned}
x(k+1) & =\Phi x(k)+\Gamma u(k), \\
y(k) & =\Delta x(k),
\end{aligned}
$$

subject to minimization of the functional

$$
J=\frac{1}{2} \sum_{m=0}^{N-1} Q(x(m))^{2}+P(u(m))^{2},
$$

where $Q \geq 0$ and $P>0$. Again, we only consider the case $Q>0$, see Remark 9. We also discard the degenerate case $\Phi=0$, since it results in zero feedback by (21). Furthermore, to ensure controllability of the system we require $\Gamma \neq 0$. Since in general $\Phi^{2} \neq 1$, it is impossible to use our previous definition (25) of a generalized Fibonacci sequence to solve the recurrence equations

$$
\begin{aligned}
\frac{L(k)}{\Phi}= & \frac{\Gamma+\frac{P}{Q} \Phi^{2} \frac{L(k+1)}{\Phi}}{\Gamma^{2}+\frac{P}{Q}+\Gamma \frac{P}{Q} \Phi^{2} \frac{L(k+1)}{\Phi}}, \\
& k=N-1, \ldots, 1,0 \\
\frac{L(N)}{\Phi}= & -\frac{Q}{P \Phi^{2}} \Gamma,
\end{aligned}
$$

and

$$
\begin{aligned}
\frac{\Gamma}{Q} R(k) & =\frac{\Gamma \frac{P}{Q}+\left(\Gamma^{2}+\frac{P}{Q} \Phi^{2}\right) \frac{\Gamma}{Q} R(k+1)}{\frac{P}{Q}+\Gamma \frac{\Gamma}{Q} R(k+1)}, \\
\frac{\Gamma}{Q} R(N) & =0 .
\end{aligned}
$$

Instead, we look for a sequence $f_{n}$ defined by the recurrence equation

$$
\begin{aligned}
& f_{n}=a_{n} f_{n-1}+b_{n} f_{n-2} \\
& f_{0}=0, f_{1}=1
\end{aligned}
$$

where we allow the coefficients $a_{n}$ and $b_{n}$ to vary with $n$. Then

$$
f_{-1}=\frac{1}{b_{1}}, f_{-2}=-\frac{a_{0}}{b_{0} b_{1}} .
$$

Moreover, we define

$$
\begin{aligned}
G_{m} & =\frac{f_{m+1}}{f_{m}}, \\
H_{m} & =\frac{f_{m-1}}{f_{m}},
\end{aligned}
$$

which implies that

$$
\begin{aligned}
G_{m} & =a_{m+1}+\frac{b_{m+1}}{G_{m-1}}, \\
G_{m+1} & =a_{m+2}+\frac{b_{m+2}}{G_{m}} .
\end{aligned}
$$

Hence

$$
\begin{aligned}
& G_{m+1}=a_{m+2}+\frac{b_{m+2}}{a_{m+1}+\frac{b_{m+1}}{G_{m-1}}} \\
& =\frac{\left(a_{m+1} a_{m+2}+b_{m+2}\right) G_{m-1}+a_{m+2} b_{m+1}}{a_{m+1} G_{m-1}+b_{m+1}}, \\
& G_{-1}=0,
\end{aligned}
$$

and similarly

$$
\begin{aligned}
H_{m+1} & =\frac{a_{m}+b_{m} H_{m-1}}{a_{m+1} a_{m}+b_{m+1}+a_{m+1} b_{m} H_{m-1}}, \\
H_{-1} & =-\frac{a_{0}}{b_{0}} .
\end{aligned}
$$

Thus we see that if we make the choice

$$
\begin{aligned}
& a_{n}=\Gamma, \text { all } n, \\
& b_{n}=\left\{\begin{array}{l}
\frac{P}{Q} \Phi^{2}, n \text { even } \\
\frac{P}{Q}, n \text { odd }
\end{array}\right.
\end{aligned}
$$

the recurrence equation (38) in the variable $\frac{\Gamma}{Q} R(\cdot)$ coincides with (40) and the recurrence equation (37) in the variable $L(\cdot) / \Phi$ coincides with (41), with the relation between indices $m$ and $k$ being

$$
m=2(N-k)-1 \text {. }
$$

This means that we can identify

$$
\begin{aligned}
\frac{\Gamma}{Q} R(k) & =G_{2(N-k)-1} \\
\frac{L(k)}{\Phi} & =H_{2(N-k)-1} .
\end{aligned}
$$

The sequences $\left(G_{n}\right)=\left(G_{-1}, G_{0}, G_{1}, \ldots\right)$ and $\left(H_{n}\right)=$ $\left(H_{-1}, H_{0}, H_{1}, \ldots\right)$ defined above can be split in the subsequences

$$
\begin{aligned}
G^{e} & =\left(G_{0}^{e}, G_{1}^{e}, G_{2}^{e}, \ldots\right), \quad H^{e}=\left(H_{0}^{e}, H_{1}^{e}, H_{2}^{e}, \ldots\right), \\
G^{o} & =\left(G_{0}^{o}, G_{1}^{o}, G_{2}^{o}, \ldots\right), \quad H^{o}=\left(H_{0}^{o}, H_{1}^{o}, H_{2}^{o}, \ldots\right),
\end{aligned}
$$

where

$$
\begin{array}{ll}
G_{m}^{e}=G_{2 m}=\frac{f_{2 m+1}}{f_{2 m}}, & H_{m}^{e}=H_{2 m}=\frac{f_{2 m-1}}{f_{2 m}}, \\
G_{m}^{o}=G_{2 m-1}=\frac{f_{2 m}}{f_{2 m-1}}, & H_{m}^{o}=H_{2 m-1}=\frac{f_{2(m-1)}}{f_{2 m-1}},
\end{array}
$$


for $m=0,1,2, \ldots$

It is worth noting that also in this case are $G_{n}$ and $H_{n}$ well defined for $n=1,2,3, \ldots$, independent of $Q_{0}$, since the corresponding bounds for the elements are

$$
\begin{aligned}
\Gamma^{2} & \leq \Gamma G_{n} \leq \Gamma^{2}+\frac{P}{Q} \max \left(1, \Phi^{2}\right), \\
\frac{1}{\Gamma^{2}+\frac{P}{Q} \max \left(1, \Phi^{2}\right)} & \leq \frac{H_{n}}{\Gamma} \leq \frac{1}{\Gamma^{2}} .
\end{aligned}
$$

This result is summarized in the main theorem of this article:

Theorem 15 Assume that $\mu_{n}$ is periodically varying as

$$
\mu_{n}=\left\{\begin{array}{l}
\Phi^{2}, n \text { even }, \\
1, n \text { odd } .
\end{array}\right.
$$

Let $f_{n}$ be the generalized Fibonacci numbers generated by

$$
\begin{aligned}
f_{n} & =\Gamma f_{n-1}+\frac{P}{Q} \mu_{n} f_{n-2} \\
f_{0} & =0, f_{1}=1
\end{aligned}
$$

and define

$$
\begin{aligned}
G_{n} & =\frac{f_{n+1}}{f_{n}}, \\
H_{n} & =\frac{f_{n-1}}{f_{n}} .
\end{aligned}
$$

Then the solution $R(N-k), k=1,2, \ldots, N$, of the Riccati equation (20) is given by

$$
R(N-k)=\frac{Q}{\Gamma} G_{2 k-1}=\frac{Q}{\Gamma} G_{k}^{o}=\frac{Q}{\Gamma} \frac{f_{2 k}}{f_{2 k-1}} .
$$

and the feedback gain $L(N-k)$ is given by

$$
L(N-k)=\Phi H_{2 k-1}=\Phi H_{k}^{o}=\Phi \frac{f_{2(k-1)}}{f_{2 k-1}} .
$$

Moreover, corresponding theorems (Theorem 11 and Theorem 12) for the state and input sequences and the value of the penalty functional hold true also in this case, since no other property from this more general definition have been used in their corresponding proofs.

Remark 16 In fact, if we know that

$$
R(N-k)=\frac{Q}{\Gamma} \frac{f_{2 k}}{f_{2 k-1}},
$$

it follows from (43) that

$$
R(N-k)=\frac{Q}{\Gamma} \frac{\Gamma f_{2 k-1}+\frac{P}{Q} \Phi^{2} f_{2(k-1)}}{f_{2 k-1}},
$$

which inserted in (21) yields

$$
\begin{aligned}
L(N-k) & =\frac{\Gamma(R(N-k)-Q)}{\Phi P} \\
& =\Phi \frac{f_{2(k-1)}}{f_{2 k-1}}=\Phi H_{2 k-1}
\end{aligned}
$$

By using the definition (42) in (39) and letting $m \rightarrow$ $\infty$, we see that

$$
\begin{aligned}
G_{m}^{e} & \rightarrow G_{\infty}^{e}, \\
G_{m}^{o} & \rightarrow G_{\infty}^{o},
\end{aligned}
$$

where

$$
\begin{aligned}
G_{\infty}^{e} & =\Gamma+\frac{\frac{P}{Q}}{G_{\infty}^{o}}, \\
G_{\infty}^{o} & =\Gamma+\frac{\frac{P}{Q} \Phi^{2}}{G_{\infty}^{e}}
\end{aligned}
$$

that is,

$$
\begin{aligned}
G_{\infty}^{e} & =\frac{1}{2 \Gamma}\left(\Gamma^{2}+\frac{P}{Q}\left(1-\Phi^{2}\right)\right. \\
& +\sqrt{\left.\left(\Gamma^{2}+\frac{P}{Q}\left(1-\Phi^{2}\right)\right)^{2}+4 \Gamma^{2} \Phi^{2} \frac{P}{Q}\right)}, \\
G_{\infty}^{o} & =\frac{1}{2 \Gamma}\left(\Gamma^{2}+\frac{P}{Q}\left(\Phi^{2}-1\right)\right. \\
& \left.+\sqrt{\left(\Gamma^{2}+\frac{P}{Q}\left(1-\Phi^{2}\right)\right)^{2}+4 \Gamma^{2} \Phi^{2} \frac{P}{Q}}\right) .
\end{aligned}
$$

Similarly, we get that

$$
\begin{aligned}
& H_{m}^{e} \rightarrow H_{\infty}^{e}=\frac{1}{G_{\infty}^{o}} \\
& H_{m}^{o} \rightarrow H_{\infty}^{o}=\frac{1}{G_{\infty}^{e}}
\end{aligned}
$$

Remark 17 Note that the limits $G_{\infty}^{e}$ and $G_{\infty}^{o}$ are independent of the initial values $f_{0}$ and $f_{1}$.

In order to find explicit Binet type expressions for $L(k)$ and $R(k)$, we need to be able to express the generalized Fibonacci numbers by some kind of recurrence equation with constant coefficents. First, we note that both $\left(G_{n}^{o}\right)$ and $\left(H_{n}^{o}\right)$ have even-indexed generalized Fibonacci numbers in the numerator and oddindexed generalized Fibonacci numbers in the denominator. We therefore try to find two recurrence equations with constant coefficients for every second generalized Fibonacci number, one for the odd-indexed subsequence and one for the even-indexed subsequence. 
Indeed, we have

$$
\begin{aligned}
f_{n+2} & =\Gamma f_{n+1}+\frac{P}{Q} f_{n} \\
& =\Gamma\left(\Gamma f_{n}+\frac{P}{Q} \Phi^{2} f_{n-1}\right)+\frac{P}{Q} f_{n} \\
& =\left(\Gamma^{2}+\frac{P}{Q}\right) f_{n}+\frac{P}{Q} \Phi^{2}\left(f_{n}-\frac{P}{Q} f_{n-2}\right) \\
& =\left(\Gamma^{2}+\frac{P}{Q}\left(\Phi^{2}+1\right)\right) f_{n}-\left(\frac{P}{Q}\right)^{2} \Phi^{2} f_{n-2},
\end{aligned}
$$

for $n$ odd and

$$
\begin{aligned}
f_{n+2} & =\Gamma f_{n+1}+\frac{P}{Q} \Phi^{2} f_{n} \\
& =\Gamma\left(\Gamma f_{n}+\frac{P}{Q} f_{n-1}\right)+\frac{P}{Q} \Phi^{2} f_{n} \\
& =\left(\Gamma^{2}+\frac{P}{Q} \Phi^{2}\right) f_{n}+\frac{P}{Q}\left(f_{n}-\frac{P}{Q} \Phi^{2} f_{n-2}\right) \\
& =\left(\Gamma^{2}+\frac{P}{Q}\left(\Phi^{2}+1\right)\right) f_{n}-\left(\frac{P}{Q}\right)^{2} \Phi^{2} f_{n-2},
\end{aligned}
$$

for $n$ even, i.e. the same recurrence relation. The corresponding initial values are

$$
\begin{aligned}
& f_{0}^{o}=f_{-1}=\frac{Q}{P}, \\
& f_{1}^{o}=f_{1}=1,
\end{aligned}
$$

for the odd-indexed subsequence $f^{o}=\left(f_{n}^{o}\right)_{n=0,1,2, \ldots}=$ $\left(f_{-1}, f_{1}, f_{3}, \ldots\right)$ and

$$
\begin{aligned}
& f_{0}^{e}=f_{-2}=-\frac{Q^{2} \Gamma}{P^{2} \Phi^{2}}, \\
& f_{1}^{e}=f_{0}=0,
\end{aligned}
$$

for the even-indexed subsequence $f^{e}=\left(f_{n}^{e}\right)_{n=0,1,2, \ldots}=$ $\left(f_{-2}, f_{0}, f_{2}, \ldots\right)$.

Now consider the sequence $\left(g_{n}\right)=\left(g_{-1}, g_{0}, g_{1}, g_{2}, \ldots\right)$ generated by

$$
\begin{aligned}
g_{n+1} & =A g_{n}+B g_{n-1}, \\
g_{-1} & =\frac{Q}{P} \\
g_{0} & =\frac{1-\Phi}{A} .
\end{aligned}
$$

Then

$$
\begin{aligned}
g_{n+2} & =A\left(A g_{n}+B g_{n-1}\right)+B g_{n} \\
& =\left(A^{2}+B\right) g_{n}+B\left(g_{n}-B g_{n-2}\right) \\
& =\left(A^{2}+2 B\right) g_{n}-B^{2} g_{n-2}, \\
g_{-1} & =\frac{Q}{P}, \\
g_{1} & =1-\Phi+B \frac{Q}{P} .
\end{aligned}
$$

If we compare this with recurrence equation (44) and its corresponding initial values (46), we see that the odd-indexed subsequence $g^{o}=\left(g_{-1}, g_{1}, g_{3}, \ldots\right)$ coincides with $f^{o}=\left(f_{-1}, f_{1}, f_{3}, \ldots\right)$ if we make the identification

$$
\begin{aligned}
A & =\frac{\sqrt{\Gamma^{4}+\Gamma^{2} \frac{P}{Q}(\Phi-1)^{2}}}{\Gamma}, \\
B & =\frac{P}{Q} \Phi .
\end{aligned}
$$

Thus we can generate the odd-indexed subsequence $f^{o}$ by taking every second element $g_{-1}, g_{1}, g_{3}, \ldots$ generated by the recurrence equation

$$
\begin{aligned}
g_{n+1} & =\frac{\sqrt{\Gamma^{4}+\Gamma^{2} \frac{P}{Q}(\Phi-1)^{2}}}{\Gamma} g_{n}+\frac{P}{Q} \Phi g_{n-1}, \\
g_{-1} & =\frac{Q}{P}, \\
g_{0} & =\frac{(1-\Phi) \Gamma}{\sqrt{\Gamma^{4}+\Gamma^{2} \frac{P}{Q}(\Phi-1)^{2}}} .
\end{aligned}
$$

By following the same idea as in the proof of the Binet formula (9), we see that we can write

$$
g_{k}=\frac{\left(\varphi_{1}+\Phi \varphi_{2}\right) \varphi_{1}^{k}-\left(\varphi_{2}+\Phi \varphi_{1}\right) \varphi_{2}^{k}}{\varphi_{1}^{2}-\varphi_{2}^{2}}
$$

where

$$
\begin{aligned}
\varphi_{1} & =\frac{A+\sqrt{A^{2}+4 B}}{2} \\
& =\frac{\sqrt{\Gamma^{4}+\Gamma^{2} \frac{P}{Q}(\Phi-1)^{2}}+\sqrt{\Gamma^{4}+\Gamma^{2} \frac{P}{Q}(\Phi+1)^{2}}}{2 \Gamma}, \\
\varphi_{2} & =\frac{A-\sqrt{A^{2}+4 B}}{2} \\
& =\frac{\sqrt{\Gamma^{4}+\Gamma^{2} \frac{P}{Q}(\Phi-1)^{2}}-\sqrt{\Gamma^{4}+\Gamma^{2} \frac{P}{Q}(\Phi+1)^{2}}}{2 \Gamma},
\end{aligned}
$$

are the roots of the corresponding characteristic equation

$$
\varphi^{2}=\frac{\sqrt{\Gamma^{4}+\Gamma^{2} \frac{P}{Q}(\Phi-1)^{2}}}{\Gamma} \varphi+\frac{P}{Q} \Phi .
$$

Hence we can conclude that

$$
\begin{gathered}
f_{k}^{o}=\quad g_{2 k-1}=\frac{\left(\varphi_{1}+\Phi \varphi_{2}\right) \varphi_{1}^{2 k-1}-\left(\varphi_{2}+\Phi \varphi_{1}\right) \varphi_{2}^{2 k-1}}{\varphi_{1}^{2}-\varphi_{2}^{2}}, \\
k=0,1,2, \ldots
\end{gathered}
$$

Similarly, we can generate the even-indexed subsequence $\left(f_{n}^{e}\right)$ by taking every second element 
$\left(h_{-2}, h_{0}, h_{2}, \ldots\right)$ generated by the recurrence equation

$$
\begin{aligned}
h_{n+1} & =\frac{\sqrt{\Gamma^{4}+\Gamma^{2} \frac{P}{Q}(\Phi-1)^{2}}}{\Gamma} h_{n}+\frac{P}{Q} \Phi h_{n-1}, \\
h_{-2} & =-\frac{Q^{2} \Gamma}{P^{2} \Phi^{2}}, \\
h_{-1} & =\frac{Q \Gamma^{2}}{P \Phi \sqrt{\Gamma^{4}+\Gamma^{2} \frac{P}{Q}(\Phi-1)^{2}}} .
\end{aligned}
$$

In this case, the corresponding Binet type formula becomes

$$
h_{k}=\Gamma \frac{\varphi_{1}^{k}-\varphi_{2}^{k}}{\varphi_{1}^{2}-\varphi_{2}^{2}}
$$

i.e. we can conclude that

$$
f_{k}^{e}=h_{2(k-1)}=\Gamma \frac{\varphi_{1}^{2(k-1)}-\varphi_{2}^{2(k-1)}}{\varphi_{1}^{2}-\varphi_{2}^{2}}, k=0,1,2, \ldots
$$

Thus we have found explicit formulae for $R(N-k)$ and $L(N-k)$ given by

$$
\begin{aligned}
& R(N-k)=\frac{Q}{\Gamma} G_{k}^{o}=\frac{Q}{\Gamma} \frac{f_{k+1}^{e}}{f_{k}^{o}} \\
& =Q \frac{\varphi_{1}^{2 k}-\varphi_{2}^{2 k}}{\left(\varphi_{1}+\Phi \varphi_{2}\right) \varphi_{1}^{2 k-1}-\left(\varphi_{2}+\Phi \varphi_{1}\right) \varphi_{2}^{2 k-1}}, \\
& L(N-k)=\Phi H_{k}^{o}=\Phi \frac{f_{k}^{e}}{f_{k}^{o}} \\
& =\Phi \Gamma \frac{\varphi_{1}^{2(k-1)}-\varphi_{2}^{2(k-1)}}{\left(\varphi_{1}+\Phi \varphi_{2}\right) \varphi_{1}^{2 k-1}-\left(\varphi_{2}+\Phi \varphi_{1}\right) \varphi_{2}^{2 k-1}}
\end{aligned}
$$

where

$$
\begin{gathered}
\varphi_{1}=\frac{\sqrt{\Gamma^{4}+\Gamma^{2} \frac{P}{Q}(\Phi-1)^{2}}+\sqrt{\Gamma^{4}+\Gamma^{2} \frac{P}{Q}(\Phi+1)^{2}}}{2 \Gamma}, \\
\varphi_{2}=\frac{\sqrt{\Gamma^{4}+\Gamma^{2} \frac{P}{Q}(\Phi-1)^{2}}-\sqrt{\Gamma^{4}+\Gamma^{2} \frac{P}{Q}(\Phi+1)^{2}}}{2 \Gamma} .
\end{gathered}
$$

Remark 18 Note that all formulae in the special case $\Phi^{2}=1$ coincide with those given in the previous section.

Remark 19 Another way to show this Binet type formula is to transform the Riccati difference equation (equivalent to eq. (38)),

$$
\begin{gathered}
R(N-k-1)=\frac{P Q+\left(Q \Gamma^{2}+P \Phi^{2}\right) R(N-k)}{P+\Gamma^{2} R(N-k)}, \\
k=0,1, \ldots, N-1,
\end{gathered}
$$

by the change of variables

$$
R(N-k)=\frac{Q \Gamma^{2}+P \Phi^{2}+P}{\Gamma^{2}} w_{k}-\frac{P}{\Gamma^{2}} .
$$

This yields the equivalent one-parameter difference equation

$$
w_{k+1}=1-\frac{\rho}{w_{k}}
$$

where

$$
\rho=\left(\frac{P \Phi}{Q \Gamma^{2}+P \Phi^{2}+P}\right)^{2} .
$$

A second change of variables

$$
w_{k}=\frac{y_{k+1}}{y_{k}}
$$

linearizes this equation to the resulting linear second order difference equation in $y_{n}$

$$
y_{k+2}-y_{k+1}+\rho y_{k}=0,
$$

which easily can be solved. For details, see e.g. the books Camouzis and Ladas (2008), Elaydi (2005) and Kulenović and Ladas (2002).

Remark 20 To handle the more general cost functional (6), one needs to replace the initial values with

$$
\begin{aligned}
& f_{0}=\frac{\Gamma}{P} Q_{0}, \\
& f_{1}=1+\frac{\Gamma^{2}}{P} Q_{0},
\end{aligned}
$$

or equivalently,

$$
\begin{aligned}
f_{-1} & =\frac{Q}{P} \\
f_{-2} & =\frac{\Gamma Q}{\Phi^{2} P^{2}}\left(Q_{0}-Q\right) .
\end{aligned}
$$

\section{Some Numerical Examples}

We will illustrate the use of generalized Fibonacci sequences in four different examples.

Example 21 Let $N=6$. We consider the process

$$
\xi(k+1)=\xi(k)+u(k), k=0,1,2, \ldots, N,
$$

under the maximization of the functional

$$
\widetilde{J}=\sum_{m=0}^{N-1} 10 \xi(m)-\frac{1}{2}(\xi(m))^{2}-\frac{1}{2}(u(m))^{2} .
$$

Moreover, we assume that we start with a value of $\xi(0)=9$. First, we see that the change of variable $x(k)=\xi(k)-10$ transforms the problem to the equivalent $L Q R$ problem

$$
x(k+1)=x(k)+u(k),
$$




\begin{tabular}{|c|r|r|r|r|r|r|}
\hline$k$ & $R(k)$ & $L(k)$ & $x(k)$ & $u(k)$ & $\xi(k)$ & $J_{k}$ \\
\hline 5 & 1 & 0 & $-\frac{1}{89} \approx-0.0112$ & 0 & 9.989 & $\frac{1}{2} \cdot \frac{1}{89^{2}}$ \\
4 & $\frac{3}{2}=1.5000$ & $\frac{1}{2}=0.5000$ & $-\frac{2}{89} \approx-0.0225$ & $\frac{1}{89} \approx 0.0112$ & 9.978 & $\frac{1}{2} \cdot \frac{2 \cdot 3}{89^{2}}$ \\
3 & $\frac{8}{5}=1.6000$ & $\frac{3}{5}=0.6000$ & $-\frac{5}{89} \approx-0.0562$ & $\frac{3}{89} \approx 0.0337$ & 9.944 & $\frac{1}{2} \cdot \frac{5 \cdot 8}{89^{2}}$ \\
2 & $\frac{21}{13} \approx 1.6154$ & $\frac{8}{13} \approx 0.6154$ & $-\frac{13}{89} \approx-0.1461$ & $\frac{8}{89} \approx 0.0899$ & 9.854 & $\frac{1}{2} \cdot \frac{13 \cdot 21}{89^{2}}$ \\
1 & $\frac{55}{34} \approx 1.6176$ & $\frac{21}{34} \approx 0.6176$ & $-\frac{34}{89} \approx-0.3820$ & $\frac{21}{89} \approx 0.2360$ & 9.618 & $\frac{1}{2} \cdot \frac{34.55}{89^{2}}$ \\
0 & $\frac{144}{89} \approx 1.6180$ & $\frac{55}{89} \approx 0.6180$ & -1 & $\frac{55}{89} \approx 0.6180$ & 9.000 & $\frac{1}{2} \cdot \frac{144}{89}$ \\
\hline
\end{tabular}

Table 1: Numerical values corresponding to Example 21.

\begin{tabular}{|r|r|r|r|r|r|r|r|r|r|r|r|r|r|}
\hline$n$ & 0 & 1 & 2 & 3 & 4 & 5 & 6 & 7 & 8 & 9 & 10 & 11 & 12 \\
\hline$f_{n}$ & 0 & 1 & 1 & 2 & 3 & 5 & 8 & 13 & 21 & 34 & 55 & 89 & 144 \\
\hline
\end{tabular}

Table 2: Fibonacci numbers corresponding to Example 21.

with initial value $x(0)=-1$, subject to minimization of the functional

$$
J=J_{0}=\frac{1}{2} \sum_{m=0}^{N-1}(x(m))^{2}+(u(m))^{2}=50-\widetilde{J} .
$$

The change of variable implies that the optimal path $\xi(k)$ is offset by 10 , i.e. $\xi(k)=x(k)+10$. Here $P=Q=1, \Phi=1$ and $\Gamma=1$, thus by using expressions (20), (21), (19), (2) and (30), we can generate Table 1. To see the relation with the sequence of Fibonacci numbers $F_{n}$, we need to consider the consecutive ratios of the numbers $F_{n}$ generated by the recurrence formula $F_{n}=F_{n-1}+F_{n-2}$. In this case, we get the familiar sequence in Table 2. Clearly, the values of $x(k), u(k)$ and $J_{k}$ all coincide with the predicted values from (28), (29) and (31). Moreover, we generate the sequences of consecutive ratios $G_{n}=\frac{F_{n+1}}{F_{n}}$ and $H_{n}=\frac{F_{n-1}}{F_{n}}$ in Table 3. As shown theoretically before, the oddindexed elements of $G_{n}$ (bold face) coincide with $R(k)$ and the odd-indexed elements of $H_{n}$ (bold face) coincide with $L(k)$, where $n=2(N-k)-1=11-2 k$, $k=0,1,2,3,4,5$.

Example 22 Let $N=5$. We consider the process $x(k+1)=-x(k)+5 u(k), k=0,1,2, \ldots, N$, with initial value $x(0)=1$, subject to minimization of the functional

$$
J=J_{0}=\frac{5}{4} \sum_{m=0}^{N-1}(x(m))^{2}+6(u(m))^{2} .
$$

Here $P=15, Q=\frac{5}{2}, \Phi=-1$ and $\Gamma=5$. Thus by using expressions (20), (21), (19), (2) and (30), we can generate Table 4. In this case, we need to find the generalized Fibonacci numbers $f_{n}$ generated by the recurrence formula $f_{n}=\Gamma f_{n-1}+\frac{P}{Q} f_{n-2}=5 f_{n-1}+6 f_{n-2}$. This gives the sequence in Table 5, resulting in the sequences of consecutive ratios $G_{n}=\frac{f_{n+1}}{f_{n}}$ and $H_{n}=\frac{f_{n-1}}{f_{n}}$ given by Table 6. Again, we note that the odd-indexed elements of $G_{n}$ multiplied with $\frac{Q}{\Gamma}=\frac{1}{2}$ (bold face) coincide with $R(k)$ and the odd-indexed elements of $H_{n}$ (bold face) multiplied with $\Phi=-1$ coincide with $L(k)$, where $n=2(N-k)-1=9-2 k, k=0,1,2,3,4$. The characteristic equation is in this case

$$
\varphi^{2}=\Gamma \varphi+\frac{P}{Q}=5 \varphi+6,
$$

with solutions

$$
\varphi_{1}=6, \quad \varphi_{2}=-1 .
$$

Thus formulae (32) and (33) give the explicit expres-

\begin{tabular}{|c|c|c|c|c|c|c|c|c|c|c|c|}
\hline$n$ & 1 & 2 & 3 & 4 & 5 & 6 & 7 & 8 & 9 & 10 & 11 \\
\hline$G_{n}$ & $\mathbf{1}$ & 2 & $\frac{\mathbf{3}}{\mathbf{2}}$ & $\frac{5}{3}$ & $\frac{\mathbf{8}}{\mathbf{5}}$ & $\frac{13}{8}$ & $\frac{\mathbf{2 1}}{\mathbf{1 3}}$ & $\frac{34}{21}$ & $\frac{\mathbf{5 5}}{\mathbf{3 4}}$ & $\frac{89}{55}$ & $\frac{\mathbf{1 4 4}}{\mathbf{8 9}}$ \\
$H_{n}$ & $\mathbf{0}$ & 1 & $\frac{\mathbf{1}}{\mathbf{2}}$ & $\frac{2}{3}$ & $\frac{\mathbf{3}}{\mathbf{5}}$ & $\frac{5}{8}$ & $\frac{\mathbf{8}}{\mathbf{1 3}}$ & $\frac{13}{21}$ & $\frac{\mathbf{2 1}}{\mathbf{3 4}}$ & $\frac{34}{55}$ & $\frac{\mathbf{5 5}}{\mathbf{8 9}}$ \\
\hline
\end{tabular}

Table 3: Ratios of consecutive numbers from Table 2. 
Byström, Lystad and Nyman, "Using Fibonacci Sequences for Solving LQR Problem and Riccati Equation"

\begin{tabular}{|c|r|r|r|r|r|r|}
\hline$k$ & $R(k)$ & $\frac{Q}{\Gamma} G_{\infty}-R(k)$ & $L(k)$ & $x(k)$ & $u(k)$ & $J_{k}$ \\
\hline 4 & $\frac{5}{2}$ & $5.0 \cdot 10^{-1}$ & 0 & $\frac{1296}{1439671} \approx 0.0009$ & & $\frac{1}{2} \cdot \frac{5 \cdot 6^{8}}{2 \cdot 1439671^{2}}$ \\
3 & $\frac{185}{62}$ & $1.6 \cdot 10^{-2}$ & $-\frac{5}{31}$ & $-\frac{6696}{1439671} \approx-0.0047$ & $-\frac{1080}{1439671} \approx-0.0008$ & $\frac{1}{2} \cdot \frac{31 \cdot 185 \cdot 6^{6}}{2 \cdot 1439671^{2}}$ \\
2 & $\frac{6665}{2222}$ & $4.5 \cdot 10^{-4}$ & $-\frac{185}{1111}$ & $\frac{39996}{1439671} \approx 0.0278$ & $\frac{6660}{1439671} \approx 0.0046$ & $\frac{1}{2} \cdot \frac{1111 \cdot 6665 \cdot 6^{4}}{2 \cdot 1439671^{2}}$ \\
1 & $\frac{239945}{79982}$ & $1.3 \cdot 10^{-5}$ & $-\frac{6665}{39991}$ & $-\frac{239946}{1439671} \approx-0.1667$ & $-\frac{39990}{1439671} \approx-0.0278$ & $\frac{1}{2} \cdot \frac{39991 \cdot 239945 \cdot 6^{2}}{2 \cdot 1439671^{2}}$ \\
0 & $\frac{8638025}{2879342}$ & $3.5 \cdot 10^{-7}$ & $-\frac{239945}{1439671}$ & 1 & $\frac{239945}{1439671} \approx 0.1667$ & $\frac{1}{2} \frac{8638025}{2 \cdot 1439671}$ \\
\hline
\end{tabular}

Table 4: Numerical values corresponding to Example 22.

\begin{tabular}{|c|r|r|r|r|r|r|r|r|r|r|r|}
\hline$n$ & 0 & 1 & 2 & 3 & 4 & 5 & 6 & 7 & 8 & 9 & 10 \\
\hline$f_{n}$ & 0 & 1 & 5 & 31 & 185 & 1111 & 6665 & 39991 & 239945 & 1439671 & 8638025 \\
\hline
\end{tabular}

Table 5: Generalized Fibonacci numbers corresponding to Example 22.

sions

$$
\begin{aligned}
R(N-k) & =\frac{Q}{\Gamma} \frac{\varphi_{1}^{2 k}-\varphi_{2}^{2 k}}{\varphi_{1}^{2 k-1}-\varphi_{2}^{2 k-1}} \\
& =\frac{1}{2} \frac{6^{2 k}-(-1)^{2 k}}{6^{2 k-1}-(-1)^{2 k-1}}=\frac{36^{k}-1}{\frac{36^{k}}{3}+2}, \\
L(N-k) & =\Phi \frac{\varphi_{1}^{2(k-1)}-\varphi_{2}^{2(k-1)}}{\varphi_{1}^{2 k-1}-\varphi_{2}^{2 k-1}} \\
& =-\frac{6^{2(k-1)}-(-1)^{2(k-1)}}{6^{2 k-1}-(-1)^{2 k-1}} \\
& =-\frac{36^{k-1}-1}{6 \cdot 36^{k-1}+1},
\end{aligned}
$$

yielding for instance

$R(1)=R(5-4)=\frac{36^{4}-1}{\frac{36^{4}}{3}+1}=\frac{1679615}{559874}=\frac{239945}{79982}$,

$L(2)=L(5-3)=-\frac{36^{2}-1}{6 \cdot 36^{2}+1}=-\frac{1295}{7777}=-\frac{185}{1111}$.

Moreover, if we denote

$$
G_{\infty}=\frac{\Gamma+\sqrt{\Gamma^{2}+4 \frac{P}{Q}}}{2}=\frac{5+\sqrt{5^{2}+4 \cdot 6}}{2}=6,
$$

we note that $R(k)$ is getting closer and closer to $\frac{Q}{\Gamma} G_{\infty}=3$ and that $L(k)$ is getting closer and closer to $\Phi \frac{1}{G_{\infty}}=-\frac{1}{6}$ as $k$ decreases. We also see that the error
$\frac{Q}{\Gamma} G_{\infty}-R(k)$ decreases approximately

$$
\frac{1}{\left(\frac{\left(\frac{P}{Q}\right)}{G_{\infty}^{2}}\right)^{2}}=6^{2}=36
$$

times for every $k$ (decreasing), as predicted by formula (16) taken two times (only odd-indexed $G_{n}$ in $R(k)$ ).

Example 23 Let $N=4$. We consider the process

$$
x(k+1)=x(k)-5 u(k), k=0,1,2, \ldots, N,
$$

with initial value $x(0)=3$, subject to minimization of the functional

$$
J=J_{0}=3(x(N))^{2}+\frac{5}{4} \sum_{m=0}^{N-1}(x(m))^{2}+6(u(m))^{2} .
$$

Here $Q_{0}=6, P=15, Q=\frac{5}{2}, \Phi=1$ and $\Gamma=-5$. Thus by using expressions (20), (21), (19), (2) and (30) with $R(N)=Q_{0}$, we can generate Table $\%$. In this case, we need to find the generalized Fibonacci numbers $f_{n}$ generated by the recurrence formula $f_{n}=\Gamma f_{n-1}+$ $\frac{P}{Q} f_{n-2}=-5 f_{n-1}+6 f_{n-2}$. Here we need to replace the initial values $f_{0}$ and $f_{1}$ by

$$
\begin{aligned}
f_{0} & =\frac{\Gamma}{P} Q_{0}=-2, \\
f_{1} & =1+\frac{\Gamma^{2}}{P} Q_{0}=11 .
\end{aligned}
$$

\begin{tabular}{|c|c|c|r|r|r|r|r|r|r|}
\hline$n$ & 1 & 2 & 3 & 4 & 5 & 6 & 7 & 8 & 9 \\
\hline$G_{n}$ & 5 & $\frac{31}{5}$ & $\frac{185}{31}$ & $\frac{1111}{185}$ & $\frac{6665}{1111}$ & $\frac{39991}{6665}$ & $\frac{239945}{39991}$ & $\frac{1439671}{239945}$ & $\frac{8638025}{1439671}$ \\
$\frac{Q}{\Gamma} G_{n}$ & $\frac{\mathbf{5}}{\mathbf{2}}$ & $\frac{31}{10}$ & $\frac{\mathbf{1 8 5}}{\mathbf{6 2}}$ & $\frac{1111}{370}$ & $\frac{\mathbf{6 6 6 5}}{\mathbf{2 2 2 2}}$ & $\frac{39991}{13330}$ & $\frac{\mathbf{2 3 9 9 4 5}}{\mathbf{7 9 9 8 2}}$ & $\frac{1439671}{479890}$ & $\frac{\mathbf{8 6 3 8 0 2 5}}{\mathbf{2 8 7 9 3 4 2}}$ \\
$H_{n}$ & $\mathbf{0}$ & $\frac{1}{5}$ & $\frac{\mathbf{5}}{\mathbf{3 1}}$ & $\frac{31}{185}$ & $\frac{\mathbf{1 8 5}}{\mathbf{1 1 1 1}}$ & $\frac{1111}{6665}$ & $\frac{\mathbf{6 6 6 5}}{\mathbf{3 9 9 9 1}}$ & $\frac{39991}{239945}$ & $\frac{\mathbf{2 3 9 9 4 5}}{\mathbf{1 4 3 9 6 7 1}}$ \\
\hline
\end{tabular}

Table 6: Ratios of consecutive numbers from Table 5. 


\begin{tabular}{|c|r|r|r|r|}
\hline$k$ & $R(k)$ & $L(k)$ & $x(k)$ & $u(k)$ \\
\hline 4 & 6 & $-\frac{7}{6} \approx-1.1667$ & $\frac{648}{519881} \approx 0.00124$ & $\frac{756}{519881} \approx 0.00145$ \\
3 & $\frac{67}{22} \approx 3.045$ & $-\frac{2}{11} \approx-0.18182$ & $\frac{7128}{519881} \approx 0.01371$ & $\frac{1296}{519881} \approx 0.00249$ \\
2 & $\frac{2407}{802} \approx 3.001$ & $-\frac{67}{401} \approx-0.16708$ & $\frac{43308}{519881} \approx 0.08330$ & $\frac{7236}{519881} \approx 0.01392$ \\
1 & $\frac{86647}{28882} \approx 3.000$ & $-\frac{2407}{14441} \approx-0.16668$ & $\frac{259938}{519881} \approx 0.50000$ & $\frac{43326}{519881} \approx 0.08334$ \\
0 & $\frac{3119287}{1039762} \approx 3.000$ & $-\frac{86647}{519881} \approx-0.16667$ & 3 & $\frac{259941}{519881} \approx 0.50000$ \\
\hline
\end{tabular}

Table 7: Numerical values corresponding to Example 23.

Moreover, the negative indexed numbers $f_{-1}$ and $f_{-2}$ become

$$
\begin{aligned}
f_{-1} & =\frac{Q}{P}=\frac{1}{6}, \\
f_{-2} & =\frac{\Gamma Q}{P^{2}}\left(Q_{0}-Q\right)=-\frac{7}{36} .
\end{aligned}
$$

This gives the sequence in Table 8, resulting in the sequences of consecutive ratios $G_{n}=\frac{f_{n+1}}{f_{n}}$ and $H_{n}=$ $\frac{f_{n-1}}{f_{n}}$ given by Table 9. Again, we note that the oddindexed elements of $G_{n}$ multiplied with $\frac{Q}{\Gamma}=-\frac{1}{2}$ (bold face) coincide with $R(k)$ and the odd-indexed elements of $H_{n}$ (bold face) multiplied with $\Phi=1$ coincide with $L(k)$, where $n=2(N-k)-1=7-2 k, k=0,1,2,3,4$. If we compare with Example 22, we see that also in this case will $R(k)$ come closer and closer to

$$
\frac{Q}{\Gamma} G_{\infty}=Q \frac{\Gamma^{2}+\sqrt{\Gamma^{4}+4 \Gamma^{2} \frac{P}{Q}}}{2}=3,
$$

independent of the initial value $Q_{0}$ (and thus also independent of $f_{0}$ and $\left.f_{1}\right)$.

Example 24 Let $N=4$. We consider the process

$$
x(k+1)=3 x(k)+5 u(k), k=0,1,2, \ldots, N,
$$

with initial value $x(0)=1$, subject to minimization of the functional

$$
J=J_{0}=\frac{5}{4} \sum_{m=0}^{N-1}(x(m))^{2}+6(u(m))^{2} .
$$

Here $P=15, Q=\frac{5}{2}, \Phi=3$ and $\Gamma=5$. Thus by using expressions (20), (21), (19), (2) and (30), we can generate Table 10. In this case, we need to find the generalized Fibonacci numbers $f_{n}$ generated by the recurrence formula $f_{n}=\Gamma f_{n-1}+\frac{P}{Q} \mu_{n} f_{n-2}=5 f_{n-1}+$ $6 \mu_{n} f_{n-2}$, where

$$
\mu_{n}=\left\{\begin{array}{c}
\Phi^{2}, n \text { even }, \quad=1+4 \cdot\left(1+(-1)^{n}\right) . \\
1, n \text { odd } .
\end{array}\right.
$$

This gives us the sequence in Table 11, resulting in the sequences of consecutive ratios $G_{n}=\frac{f_{n+1}}{f_{n}}$ and $H_{n}=\frac{f_{n-1}}{f_{n}}$ given by Table 12. As before, we note that the odd-indexed elements of $G_{n}$ (bold face) multiplied with $\frac{Q}{\Gamma}=\frac{1}{2}$ coincide with $R(k)$ and the odd-indexed elements of $H_{n}$ multiplied with $\Phi=3$ (bold face) coincide with $L(k)$, where $n=2(N-k)-1=7-2 k$, $k=0,1,2,3$.

Another method to find $G_{n}$ and $H_{n}$ (using recurrence equations with constant coefficients) is to take every second element $g_{-1}, g_{1}, g_{3}, \ldots$ generated by the recurrence equation

$$
\begin{aligned}
g_{n+1} & =\frac{\sqrt{\Gamma^{4}+\Gamma^{2} \frac{P}{Q}(\Phi-1)^{2}}}{\Gamma} g_{n}+\frac{P}{Q} \Phi g_{n-1} \\
& =7 g_{n}+18 g_{n-1} \\
g_{-1} & =\frac{Q}{P}=\frac{1}{6}, \\
g_{0} & =\frac{(1-\Phi) \Gamma}{\sqrt{\Gamma^{4}+\Gamma^{2} \frac{P}{Q}(\Phi-1)^{2}}}=-\frac{2}{7},
\end{aligned}
$$

and every second element $\left(h_{-2}, h_{0}, h_{2}, \ldots\right)$ generated by the recurrence equation

$$
\begin{aligned}
h_{n+1} & =\frac{\sqrt{\Gamma^{4}+\Gamma^{2} \frac{P}{Q}(\Phi-1)^{2}}}{\Gamma} h_{n}+\frac{P}{Q} \Phi h_{n-1} \\
& =7 h_{n}+18 h_{n-1}, \\
h_{-2} & =-\frac{Q^{2} \Gamma}{P^{2} \Phi^{2}}=-\frac{5}{324}, \\
h_{-1} & =\frac{Q \Gamma^{2}}{P \Phi \sqrt{\Gamma^{4}+\Gamma^{2} \frac{P}{Q}(\Phi-1)^{2}}}=\frac{5}{126} .
\end{aligned}
$$

Then the odd-indexed elements of $\left(g_{n}\right)$ (bold face) coincide with the odd-indexed elements of $\left(f_{n}\right)$ and the even-indexed elements of $\left(h_{n}\right)$ (bold face) coincide with the even-indexed elements of $\left(f_{n}\right)$, see Table 13. These two subsequences form the basis of the Binet type for- 
Byström, Lystad and Nyman, "Using Fibonacci Sequences for Solving LQR Problem and Riccati Equation"

\begin{tabular}{|r|r|r|r|r|r|r|r|r|r|r|r|}
\hline$n$ & -2 & -1 & 0 & 1 & 2 & 3 & 4 & 5 & 6 & 7 & 8 \\
\hline$f_{n}$ & $-\frac{7}{36}$ & $\frac{1}{6}$ & -2 & 11 & -67 & 401 & -2407 & 14441 & -86647 & 519881 & -3119287 \\
\hline
\end{tabular}

Table 8: Generalized Fibonacci numbers corresponding to Example 23.

\begin{tabular}{|r|r|r|r|r|r|r|r|r|r|}
\hline$n$ & -1 & 0 & 1 & 2 & 3 & 4 & 5 & 6 & 7 \\
\hline$G_{n}$ & -12 & $-\frac{11}{2}$ & $-\frac{67}{11}$ & $-\frac{401}{67}$ & $-\frac{2407}{401}$ & $-\frac{14441}{2407}$ & $-\frac{86647}{14441}$ & $-\frac{519881}{86647}$ & $-\frac{3119287}{519881}$ \\
$\frac{Q}{\Gamma} G_{n}$ & $\mathbf{6}$ & $\frac{11}{4}$ & $\frac{\mathbf{6 7}}{\mathbf{2 2}}$ & $\frac{401}{134}$ & $\frac{\mathbf{2 4 0 7}}{\mathbf{8 0 2}}$ & $\frac{14441}{4814}$ & $\frac{\mathbf{8 6 6 4 7}}{\mathbf{2 8 8 8 2}}$ & $\frac{519881}{173294}$ & $\frac{\mathbf{3 1 1 9 2 8 7}}{\mathbf{1 0 3 9 7 6 2}}$ \\
$H_{n}$ & $-\frac{\mathbf{7}}{\mathbf{6}}$ & $-\frac{1}{6}$ & $-\frac{\mathbf{2}}{\mathbf{1 1}}$ & $-\frac{11}{67}$ & $-\frac{\mathbf{6 7}}{\mathbf{4 0 1}}$ & $-\frac{401}{2407}$ & $-\frac{\mathbf{2 4 0 7}}{\mathbf{1 4 4 4 1}}$ & $-\frac{14441}{86647}$ & $-\frac{\mathbf{8 6 6 4 7}}{\mathbf{5 1 9 8 8 1}}$ \\
\hline
\end{tabular}

Table 9: Ratios of consecutive numbers from Table 8.

mulae (48) and (49), which in this case become

$$
\begin{aligned}
& R(N-k) \\
& =Q \frac{\varphi_{1}^{2 k}-\varphi_{2}^{2 k}}{\left(\varphi_{1}+\Phi \varphi_{2}\right) \varphi_{1}^{2 k-1}-\left(\varphi_{2}+\Phi \varphi_{1}\right) \varphi_{2}^{2 k-1}} \\
& =\frac{5}{2} \frac{81^{k}-4^{k}}{27 \cdot 81^{k-1}+50 \cdot 4^{k-1}}, \\
& L(N-k) \\
& =\Phi \Gamma \frac{\varphi_{1}^{2(k-1)}-\varphi_{2}^{2(k-1)}}{\left(\varphi_{1}+\Phi \varphi_{2}\right) \varphi_{1}^{2 k-1}-\left(\varphi_{2}+\Phi \varphi_{1}\right) \varphi_{2}^{2 k-1}} \\
& =15 \frac{81^{k-1}-4^{k-1}}{27 \cdot 81^{k-1}+50 \cdot 4^{k-1}},
\end{aligned}
$$

since

$$
\begin{aligned}
& \varphi_{1}=\frac{\sqrt{\Gamma^{4}+\Gamma^{2} \frac{P}{Q}(\Phi-1)^{2}}+\sqrt{\Gamma^{4}+\Gamma^{2} \frac{P}{Q}(\Phi+1)^{2}}}{2 \Gamma} \\
& =9, \\
& \varphi_{2}=\frac{\sqrt{\Gamma^{4}+\Gamma^{2} \frac{P}{Q}(\Phi-1)^{2}}-\sqrt{\Gamma^{4}+\Gamma^{2} \frac{P}{Q}(\Phi+1)^{2}}}{2 \Gamma} \\
& =-2 .
\end{aligned}
$$

This gives for instance

$$
R(2)=R(4-2)=\frac{5}{2} \frac{81^{2}-4^{2}}{27 \cdot 81+50 \cdot 4}=\frac{425}{62}
$$

and

$$
L(1)=L(4-3)=15 \frac{81^{2}-4^{2}}{27 \cdot 81^{2}+50 \cdot 4^{2}}=\frac{1275}{2311} .
$$

Finally, we note that $R(k)$ is getting closer and closer to

$$
\begin{aligned}
\frac{Q}{\Gamma} G_{\infty}^{o} & =\frac{Q}{2 \Gamma^{2}}\left(\Gamma^{2}+\frac{P}{Q}\left(\Phi^{2}-1\right)\right. \\
& \left.+\sqrt{\left(\Gamma^{2}+\frac{P}{Q}\left(1-\Phi^{2}\right)\right)^{2}+4 \Gamma^{2} \Phi^{2} \frac{P}{Q}}\right)=\frac{15}{2}
\end{aligned}
$$

and that $L(k)$ is getting closer and closer to

$$
\begin{aligned}
\Phi H_{\infty}^{o} & =\frac{\Phi}{G_{\infty}^{e}}=2 \Gamma \Phi /\left(\Gamma^{2}+\frac{P}{Q}\left(1-\Phi^{2}\right)\right. \\
& \left.+\sqrt{\left(\Gamma^{2}+\frac{P}{Q}\left(1-\Phi^{2}\right)\right)^{2}+4 \Gamma^{2} \Phi^{2} \frac{P}{Q}}\right)=\frac{5}{9} .
\end{aligned}
$$

as $k$ decreases.

\begin{tabular}{|c|r|r|r|r|r|}
\hline$k$ & $R(k)$ & $L(k)$ & $x(k)$ & $u(k)$ & $J_{k}$ \\
\hline 3 & $\frac{5}{2}=2.5$ & 0 & $\frac{5832}{186391} \approx 0.0313$ & & $\frac{1}{2} \cdot \frac{5 \cdot 18^{6}}{2 \cdot 186391^{2}}$ \\
2 & $\frac{425}{62} \approx 6.8548$ & $\frac{15}{31} \approx 0.48387$ & $\frac{10044}{186391} \approx 0.0539$ & $-\frac{4860}{186391} \approx-0.0261$ & $\frac{1}{2} \cdot \frac{31 \cdot 425 \cdot 18^{4}}{2 \cdot 186391^{2}}$ \\
1 & $\frac{34505}{4622} \approx 7.4654$ & $\frac{1275}{2311} \approx 0.55171$ & $\frac{41598}{186391} \approx 0.22318$ & $-\frac{22950}{186391} \approx-0.12313$ & $\frac{1}{2} \cdot \frac{2311 \cdot 34505 \cdot 18^{2}}{2 \cdot 186391^{2}}$ \\
0 & $\frac{2795225}{372782} \approx 7.4983$ & $\frac{103515}{186391} \approx 0.55536$ & 1 & $-\frac{103515}{186391} \approx-0.55536$ & $\frac{1}{2} \cdot \frac{2795225}{2 \cdot 186391}$ \\
\hline
\end{tabular}

Table 10: Numerical values corresponding to Example 24.

\begin{tabular}{|r|r|r|r|r|r|r|r|r|r|}
\hline$n$ & 0 & 1 & 2 & 3 & 4 & 5 & 6 & 7 & 8 \\
\hline$f_{n}$ & 0 & 1 & 5 & 31 & 425 & 2311 & 34505 & 186391 & 2795225 \\
\hline
\end{tabular}

Table 11: Generalized Fibonacci numbers corresponding to Example 24. 


\begin{tabular}{|c|c|c|r|r|r|r|r|}
\hline$n$ & 1 & 2 & 3 & 4 & 5 & 6 & 7 \\
\hline$G_{n}$ & 5 & $\frac{31}{5}$ & $\frac{425}{31}$ & $\frac{2311}{425}$ & $\frac{34505}{2311}$ & $\frac{186391}{34505}$ & $\frac{2795225}{186391}$ \\
$\frac{Q}{\Gamma} G_{n}$ & $\frac{\mathbf{5}}{\mathbf{2}}$ & $\frac{31}{10}$ & $\frac{\mathbf{4 2 5}}{\mathbf{6 2}}$ & $\frac{2311}{850}$ & $\frac{\mathbf{3 4 5 0 5}}{\mathbf{4 6 2 2}}$ & $\frac{186391}{69010}$ & $\frac{\mathbf{2 7 9 5 2 2 5}}{\mathbf{3 7 2 7 8 2}}$ \\
$H_{n}$ & 0 & $\frac{1}{5}$ & $\frac{5}{31}$ & $\frac{31}{425}$ & $\frac{425}{2311}$ & $\frac{2311}{34505}$ & $\frac{34505}{186391}$ \\
$\Phi H_{n}$ & $\mathbf{0}$ & $\frac{3}{5}$ & $\frac{\mathbf{1 5}}{\mathbf{3 1}}$ & $\frac{93}{425}$ & $\frac{\mathbf{1 2 7 5}}{\mathbf{2 3 1 1}}$ & $\frac{6933}{34505}$ & $\frac{\mathbf{1 0 3 5 1 5}}{\mathbf{1 8 6 3 9 1}}$ \\
\hline
\end{tabular}

Table 12: Ratios of consecutive numbers from Table 11.

\begin{tabular}{|c|c|c|c|c|c|c|c|c|c|c|c|}
\hline$n$ & -2 & -1 & 0 & 1 & 2 & 3 & 4 & 5 & 6 & 7 & 8 \\
\hline$g_{n}$ & & $\frac{1}{6}$ & $-\frac{2}{7}$ & 1 & $\frac{13}{7}$ & 31 & $\frac{1753}{7}$ & 2311 & $\frac{144753}{7}$ & 186391 & $\frac{11739433}{7}$ \\
\hline$h_{n}$ & $-\frac{5}{324}$ & $\frac{5}{126}$ & 0 & $\frac{5}{7}$ & 5 & $\frac{335}{7}$ & 425 & $\frac{26855}{7}$ & 34505 & $\frac{2174135}{7}$ & 2795225 \\
\hline
\end{tabular}

Table 13: Another method to find generalized Fibonacci numbers corresponding to Example 24.

\section{Discussion and Conclusions}

In this article, we found an efficient way to solve onedimensional LQR-problems. We showed that to each specific problem, there is a corresponding Fibonacci type sequence in terms of which the solution can be written. More precisely, it turns out that the solution of the corresponding Riccati equation, the feedback gain, the controller state, the input and the subsequent penalty functional values all can be expressed as ratios of subsequent values generated by this Fibonacci type sequence. Moreover, this way of solving the LQR problem naturally implies that we can find direct explicit formulae (so called Binet type formulae) that computes the $k^{t h}$ desired value of some property, without knowledge of previous values. The article is concluded by showing the use of the theory and the formulae in four separate examples of increasing complexity.

\section{References}

Balchen, J. Reguleringsteknikk. Tapir, Trondheim, 1977.

Benavoli, A., Chisci, L., and Farina, A. Fibonacci sequence, golden section, kalman filter and optimal control. Signal Processing, 2009. 89(8):1483-1488. doi:10.1016/j.sigpro.2009.02.003.

Camouzis, E. and Ladas, G. Dynamics of Third Order Rational Difference Equations with Open Problems and Conjectures. Chapman \& Hall / CRC, Boca Raton, 2008.

Capponi, A., Farina, A., and Pilotto, C. Expressing stochastic filters via number sequences. Signal Processing, $2010 . \quad$ 90(7):2124-2132. doi:10.1016/j.sigpro.2010.01.011.

Dickson, L. E. History of the Theory of Numbers, Volume I: Divisibility and Primality. The American Mathematical Society, 2002.
Dunlap, R. The Golden Ratio and Fibonacci Numbers. World Scientific, Singapore, 2003.

Elaydi, S. An Introduction to Difference Equations (3rd ed.). Springer, New York, 2005.

Huntley, H. E. The Divine Proportion: a Study in Mathematical Beauty. Dover Publications, Mineola, 1970.

Kulenović, M. R. S. and Ladas, G. Dynamics of Second Order Rational Difference Equations: With open problems and conjectures. Chapman \& Hall / CRC, Boca Raton, 2002.

Kwakernaak, H. and Sivan, R. Linear optimal control systems. Wiley-Interscience, New York-LondonSydney, 1972.

Lang, W. Riccati meets fibonacci. The Fibonacci Quarterly, 2004. 42(3):231-244.

Lewis, F. L. and Syrmos, V. L. Optimal control (2nd Ed.). John Wiley \& Sons Inc., New York, 1995.

Livio, M. The Golden Ratio: The Story of PHI, the World's Most Astonishing Number. Broadway Books, 2002.

Ribenboim, P. My Numbers, My Friends: Popular Lectures on Number Theory. Springer Verlag, New York, 2000 .

Walser, H. The Golden Section. The Mathematical Association of America, 2001.

Åström, K. and Wittenmark, B. Computer-Controlled Systems: Theory and Design. Prentice Hall, 1996. 